\title{
THE AEROSPACE CORPORATION
}

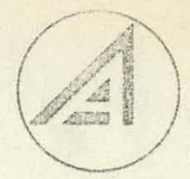

Post Office Box 92957, Los Angeles, California 90009, Telephone: (213) 648-5000

7769.79.MGH-045

10 Apri1 1979

Mr. Paul Lombardi

Chief, Vehicle Systems Section

Transportation Energy Conservation Division

U. S. Department of Energy

20 Massachusetts Avenue

Washington, D. C. 20545

Dear Mr. Lombardi:

Enclosed are seven copies of the eighth monthly progress report on our contract for "Technical Analysis Support for Transportation

Energy Conservation Division of DOE." This report covers the period 1 March through 31 March 1979.

If you have any questions about this report, please do not hesitate to call me, Wolfgang Roessler (213) 648-5550, or Toru Iura (301) 428-2754, at any time.

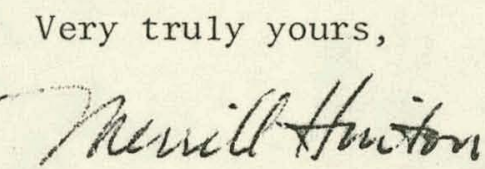

Merril1 G. Hinton

Principal Director

Mobile Systems Directorate

Telephone: (213) $648-\% 110$

MGH: pm

Enclosures, as noted

cc - William Lambert

$\mathrm{bc}-$

L. Forrest

R. T. Hall

$\mathrm{T}$. Iura

J. Meltzer

D.R. Orozco

W.U. Roessler

D. Rubio 


\section{DISCLAIMER}

This report was prepared as an account of work sponsored by an agency of the United States Government. Neither the United States Government nor any agency Thereof, nor any of their employees, makes any warranty, express or implied, or assumes any legal liability or responsibility for the accuracy, completeness, or usefulness of any information, apparatus, product, or process disclosed, or represents that its use would not infringe privately owned rights. Reference herein to any specific commercial product, process, or service by trade name, trademark, manufacturer, or otherwise does not necessarily constitute or imply its endorsement, recommendation, or favoring by the United States Government or any agency thereof. The views and opinions of authors expressed herein do not necessarily state or reflect those of the United States Government or any agency thereof. 


\section{DISCLAIMER}

Portions of this document may be illegible in electronic image products. Images are produced from the best available original document. 


\section{EIGHTH PROGRESS REPORT}

(for March 1979)

TECHNICAL ANALYSIS SUPPORT FOR

TRANSPORTATION ENERGY CONSERVATION DIVISION OF DOE

10 April 1979

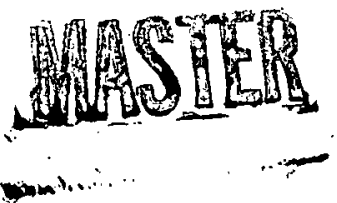

Contract No. EM-78-C-03-2184

Prepared by

Mubile Systems Directorate.

Eastern Technical Division

THE AEROSPACE CORPORATION

EI Segundo, California

for the

Transportation Energy Conservation Division

U. S. DEPARTMENT OF ENERGY

Washington, D. C .

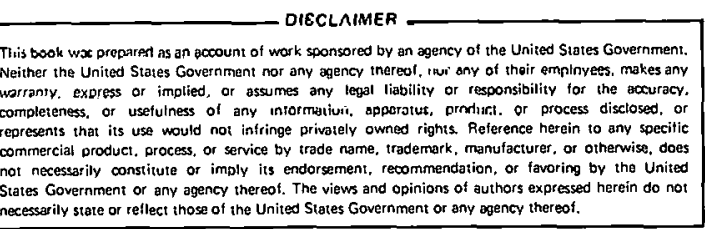

DISTRIBUTION OF THIS DOCUMEQT IS UNLIMITED 


\title{
EIGHTH PROGRESS REPORT
}

(for March 1979)

\begin{abstract}
TECHNICAL ANALYSIS SUPPORT FOR TRANSPORTATION ENERGY CONSERVATION DIVISION OF DOE
\end{abstract}

1.

INTRODUCTION

The Aerospace Corporation is providing technical analysis support services to the DOE Transportation Energy Conservation (TEC) Division under Contract No. EM-73-C-03-2184. These services were initiated on June 15, 1978, under an advance agreement; the contract was signed by DOE on August 17, 1978 .

The nature of the services being provided is similar to that of a previous 30-month support services contract between Aerospace and DOE/TEC (Contract No. EY-76-C-03-1101, Project Agreement No. 3); in several instances the work to be done is a direct continuation of activities initiated under the previous contract.

The period of performance under this contract commenced on June 15, 1978 and expires on June 14, 1979. DOE has the right to authorize an optional additional year of effort which could extend the contract period to June 14,1980 . The contract is being incrementally funded; $\$ 1,060,000$ is the amount presently obligated. The obligation limit for the first year of effort. (through June 14,1979 ) is $\$ 1,488,785$.

The following sections (1) present the status of achieved progress through the period ending 31 March 1979, and (2) summarize the expenditure status. Historical information concerning task assignments and reporting activities is provided in Appendices $A$ and $B$. $2 \quad$ ORGANIZATION OF SUPPORT EFFORT

Five Branches within DOE/TEC have presently committed funding support to the contract; they include: 
1. Heat Engine Systems (G. Thur)

2. Alternative Fuels Utilization (E. Ecklund)

3. Nonhighway Transport Systems (R. Alpaugh)

4. New Concepts Evaluation (M. Starr)

5. Electric and Hybrid Vehicle $R$ \& $D$ (R. Kirk and K. Barber)

\begin{tabular}{|c|c|c|c|}
\hline$\$ 400,000$ & $\$ 500,000$ & $\$$ & 900,000 \\
\hline 175,000 & 75,000 & & 250,000 \\
\hline 120,000 & 100,000 & & 220,000 \\
\hline 60,000 & - & & 60,000 \\
\hline 305,000 & 265,000 & & 570,000 \\
\hline 0 & $\$ 940,000$ & & 000,000 \\
\hline
\end{tabular}

A sixth Branch, Technology Assessment and Implementation, may elect to commit funds at a later date. In addition, it is contemplated that it will be necessary to support certain TEC-wide program documentation efforts.

To provide for control and tracking of expenditures and efforts for each of the above Branches and Division activities, a series of Job Orders (JO's) has been initially selected and assigned to current and prospective support needs. They are summarized in Table 1. An historical description of the work efforts assigned during this contract for the above activities is presented in Appendix A.

3. PROGRESS FOR THIS REPORTING PERIOD

Brief summaries of status and progress are given below for those support activities in progress through March 31, 1979.

\section{$3.1 \quad$ Heat Engine Systems}

$3.1 .1 \quad$ Autumulive Technology $\Lambda s s e s s m e n t$ Study (.Tn 7751)

Objective: To assist TEC in preparing the annual report to Congress required by Automotive Propulsion Research and Development Act of 1978 (Title III of Public Law 95-238). Requires technology assessments, planning, and report writing and coordination.

Status: Comments and suggestions pertaining to the format and contents of the Annual Report were developed and presented to the DOE/NASA Automotive Heat Engine Program Management Review Meeting on June 17, 1978. General agreement was reached on a plan whereby Aerospace and NASA/Lewis would prepare initial 
inputs. A rough draft strawman document was propared by Aerospace and transmitted to Mr. Lombardi on 1 November 1978 for his review and approval. DOE's review comments were discussed in a number of meetings and telecommunications with Mr. Lombardi, Mr. Thur, and Mr. Brogan. The final version of the report was transmitted to $\mathrm{Mr}$. Lombardi on 27 February 1979.

\subsubsection{Technical Responses to Inquiries (JO 7752)}

Objective: To provide quick-response support by (1) reviewing inquiries submitted to the Office of Highway Systems, and (2) preparing technical inputs for letter responses to inventors, Congressional offices, and concerned citizens. Status: During the month of March, technical responses were prepared for 30 inquiries which were received by the Office of Highway Systems.

3.1.3 Highway Systems Analysis Support (JO 7753)

3.1.3.1. Fuel Consumption and Emission Characteristics of Stratified Charge Engines

Objective: To assess the technological status of stratified charge engines and provide a basis for predicting the fuel economy potential of these engine/ vehicle systems as affected by increasingly stringent $\mathrm{NO}_{\mathrm{x}}$ and $\mathrm{HC}$ emission regulations. Of particular interest is the projected fuel economy that might be achieved with these engines at the statutory $\mathrm{NO}_{\mathrm{x}}$ level of $0.4 \mathrm{gram} / \mathrm{mile}$. Status: This study, which was initiated during the previous contract, involves the analysis of the emissions and fuel economy characteristics of Ford's PROCO and Texaco's TCCS open-chamber stratified charge engines, and the effects of $\mathrm{NO}_{x}$ control level and inertia weight variations on fuel economy of vehicles equipped with these engines. A draft report was completed which is currently undergoing in-house review, preparatory to transmittal of the report to DOE.

\subsubsection{Summary of Diesel-related Work in Areas of Technology Development and Health Effects}

Objective: To summarize all diesel-related work in the areas of technology development and health effects currently in progress by industry and governmental agencies. To identify specific areas which are currently not adequately 
covered by ongoing or projected prograns, and which therefore are considered to be candidates for DOE support.

Status: At the request of $\mathrm{Mr}$. Themak, EPA's draft, "Regulatory Analysis Report on Light-duty Diesel Particulate Regulations," was reviewed, and an analysis was performed of diesel emission control system performance, with particular emphasis on the tradeoffs between $\mathrm{HC}$, $\mathrm{NO}_{\mathrm{x}}$, particulates, and fuel economy. A brief summary report was submitted to Mr. Themak on 2 March 1979.

\subsubsection{Heat Engine Thermal Efficiency}

Objective: To determine theoretical and actual air cycle and fuel-air cycle efficiencies for automotive heat engines.

Status: Theoretical and brake thermal efficiencies were computed for Otto, diesel, Brayton, and Stirling cycle engines as a function of many parameters, including compression ratio, air-fuel ratio, turbine inlet temperature, Stirling heater head temperature, and regenerator effectiveness. The computed efficiencies at the best operating point of the engine and over the Federal Driving Cycle were compared with actual values determined from measured vehicle fuel economy data. Results of this analysis were transmitted to Mr. Brogan on 16 March 1979. A final report is in preparation.

\subsubsection{Engine-Fue1-Refinery System Tradeoff}

Objective: To determine transportation mileage and crude oil savings related to the production and utilization of alternate fuels.

Status: $\quad$ Crude oil utilization efficiency expressed in terms of vehicle miles driven per barrel of crude oil, was determined for a number of scenarios involving the increased use of diesel engines, as well as the introduction of gas turbines, Stirling engines, and open-chamber stratified charge engines using wide-cut fuels in place of gasoline or diesel fuel. Initial study results were transmitted to Mr.Brogan on 23 March 1979.

\subsubsection{Evaluation of Unsolicited Proposals and Reports}

Objective: To perform technical evaluations of unsolicited proposals and reports submitted to the Office of Highway Systems. 
Status: At the request of $\mathrm{Mr}$. Lombardi, three unsolicited proposals were reviewed during this report period. It was recommended that DOE not support the development of the proposed concepts.

One proposal, entitled "Pentagonal Electromagnetic Engine," involves the development of an electric motor composed of five solenoids arranged in a circle and operating a connecting rod/crankshaft mechanism identical in concept to a radial piston engine. The use of solenoids and a complicated mechanical linkage to provide a rotary motor was judged to be much less efficient than the conventional DC electrical motor.

The second proposal, entitled "Gearbine Rotary Engine," is concerned with a Brayton cycle type engine consisting of a multistage gear pump for air compression, with the same gear units being used for expansion of the combustion gas. Because of its inferior efficiency and large size relative to conventional gas turbines and Otto cycle engines, the proposed engine has no fuel conservation potential.

The third proposal involves the development of a dual-cycle heat engine, consisting of a Rankine cycle unit and a refrigeration unit. The refrigerant is supposed to absorb the heat of vaporization of the Rankine cycle, returning the heat plus the compression heat to the Rankine cycle. The system as proposed violates the second law of thermodynamics, and has no fuel conservation potential.

At the request of $\mathrm{Mr}$. Alpaugh, a review is being made of an unsolicited proposal and background material on wind-powered cargo ships. The proposer forecasts fuel savings up to 85 percent relative to conventional cargo ships at the expense of somewhat longer trip times.

\subsubsection{Development of Vehicle Systems Program Activity Displays}

Objective: To define and develop visual techniques to display and monitor the content and activity level of the Vehicle Systems Program.

Status: Data were collected on ten programs for display on the DOE status chart. The ten programs are Advanced Transmissions, Chrysler Gas Turbine Vehicles, Stirling Engine Vehicles, Gas Turbine Transit Bus Demonstration, 
Controlled Speed Accessory Drive Demonstration, Truck Bottoming Cycle Program, Turbocompound Diese1 Truck, Ceramic Gas Turbine Truck, Hydrogen Engine Vehicle, and Student Competitions on Relevant Engineering. Preliminary conceptual activity summary sheets were prepared for each project and provided to P. Lombardi. Based on his review and the comments of A. Chesnes, the next level of activity was focused on a geographic orientation status board for top level tracking of project status. This status board is scheduled for completion by 20 April.

\subsubsection{Research Needs for Automotive Engines}

Objective: To identify basic and applied research needs in the areas of intermittent and continuous combustion automotive heat engines, and to outline specific research programs aimed at the achievement of significant improvements in automobile fuel economy.

Status: At the request of $\mathrm{Mr}$. Brogan, a list of research needs was prepared for various categories of advanced automotive power systems. The Conference on Basic Research Directions for Advanced Automotive Technology, held on February 13 and 14, 1979 in Boston, Massachusetts, was attended, and quickresponse support was provided at that meeting to both the Engine and Fuels/ Powertrain Systems Panels, assisting these panels in the preparation of summary reports to the Secretary of Transportation.

\subsection{Alternative Fuels Utilization}

\subsubsection{Distillery Impact Study (JO 7756)}

Objective: To determine the role the distilling industry can fill in producing alcohol for fuel use; to determine the technological, economic, institutional, and environmental characteristics of the fermentation industries producing industrial ethanol and distilled spirits.

Status: $\quad$ The work plan for this study was defined during the previous contract and submitted to TEC on March 30, 1978. During the July 30 to August 4 time period, visits were made and discussions held to acquire data needed for further analysis. Companies visited included: Kentucky Distillers Association, Frankfort, Kentucky; 01d Grand Dad, Frankfort, Kentucky; Midwest 
Solvents, Atchison, Kansas; Milbrew, Milwaukee, Wisconsin; and Georgia-Pacific Corporation, Bellingham, Washington.

A rough draft of the final report was completed in mid-December and forwarded to E. E. Ecklund for review. His review comments were received in late January. The final report (ATR-79(7756)-1) was published and forwarded to Mr. Ecklund on 30 March.

3.2.2 Fuel Economy Improvement Potential Study (J0 7758)

Objective: To determine the combustion efficiency potential of spark ignition engines änd the potential impact of fuel modifications. Phase I involves the analysis of combustion losses of late-model engines; Phase II involves theoretical analyses of combustion inefficiencies as affected by variations in fuel heat release rate.

Status: The study of engine efficiency losses, as represented by $\mathrm{HC}$ and CO exhaust emissions, was completed during this report period. Vehicle simulation (VEHSIM) computer program calculations were performed for eight 1977 and six 1978 model year spark ignition and diesel engine/vehicle configurations, considering both raw engine and catalyst emissions. Combustion efficiency results obtained from these vehicle simulations over the urban driving cycle, and from combustion efficiency maps previously calculated, were summarized in two interim reports transmitted to Dr. Fleming on 15 November 1978 and 30 March 1979.

The Phase II final report, which analyzes the effects of combustion rate on engine efficiency, was transmitted to Dr. Fleming on 9 February 1979.

\subsubsection{Alternative Fuels Analysis Support (JO 7759)}

\subsubsection{Hydrogen Powered vs. Electric Automobiles}

Objective: To compare the costs and benefits of the generation of hydrogen and its use as fuel in a spark ignition engine versus the direct use of electric energy in battery-operated vehicles.

Status: The study has been completed and documented in a final draft report consisting of an executive summary and separate sections on each of the four principal study tasks: (1) hydrogen production, (2) hydrogen vehicle 
assessment, (3) electric vehicle assessment, and (4) technical and economic comparison of hydrogen and battery-powered vehicles. The draft report, which has been reviewed by nine outside organizations, has been transmitted to DOE for review, and approval for publication has been received from Mr. Ecklund. The report was turned over to the publications group in February for final editing and printing.

\subsubsection{Project Plan for Reliability Fleet Testing of Alcohol/Gasoline Blends}

Objective: To prepare a Project Plan which defines all aspects of the requirements for adequately demonstrating the in-use reliability of alcohol/gasoline blends via fleet testing.

Status: An initial version of the project plan was completed and transmitted to E. E. Ecklund on July 31, 1978. Review comments were received and the document was revised accordingly. The second version was submitted in mid-September and further revised in mid-October. A meeting was held on December 6, 1978 to review the plans made to date. Based on that review, the CRC meeting in Detroit on December 12 was attended to get their inputs to and comments on the plan. Efforts were initiated during the third week in December to secure the services of SWRI to assist in planning the fleet testing activities. SWRI was issued a purchase order in mid-January and has commenced the required task.

The CRC Alternate Automotive Fuels Group meeting was attended in Atlanta, Georgia on February 14. The principal discussion focused on subgroup reports concerning analytical procedures, fuel selection, and vehicle selection criteria to be recommended fur alculiol/gasoline blend usc.

The following day, Mr. E. Eugene Ecklund convened a meeting of CRC members and other interested parties to review the Fleet Selection Criteria Document prepared by SWRI and Aerospace. This document will be revised according to the comments received.

A March 30, 1979 revision of the Project Plan was completed which incorporated the results of all the foregoing meetings and discussions. A presentation was prepared to outline the program at the Highway Vehicle Systems Contractors' Coordination Meeting in Dearborn, Michigan, April 24-16,-1979. 


\subsubsection{Nonhighway Transport Systems}

3.2.4.1 Commercial Aviation Nonhardware Energy Conservation Strategies (JO 7761)

Objective: To examine commercial aviation operational policies and fuel conservation strategies and identify those that are the most beneficial and deserving of DOE support.

Status: During the month of March, letters were received from Continental and Alaska Airlines delineating the incremental costs which they would incur and charge, as subcontractors to Aerospace, for their participation in a program intended to demonstrate reduced fuel consumption of commercial airliners through improved airframe maintenance. Subsequently, work statements were drafted describing the airlines' tasks in the planned program. The work statement and airline cost estimates are intended to be used as a basis for negotiating separate subcontracts wi.th Continental and Alaska.

At the request of $\mathrm{Mr}$. Alpaugh, a presentation was given to the FAA on 16 March 1979 on the recently completed Aerospace study of Commercial Aviation Operational Energy Conservation Strategies. In preparation for this presentation, an analysis of the relative contribution of load factor changes and the contribution of the implementation of conservation strategies to year-to-year variations in revenue ton miles per gallon (RTMPG) performance was formulated. Results of the analysis show that only about 1 percent of a 16 percent improvement in RTMPG between 1972 and 1975 can be attributed to conservation efforts, while the remaining 15 percent improvement is related to load factor changes. A recent FAA report attributes the entire 16 percent improvement in RTMPG to implementation of conservation strategies.

\subsubsection{New Concepts Evaluation (JO 7764)}

Objective: To provide analyses and technical support to facilitate DOE's evaluation of specific proposals received by DOE.

Status: No proposal evaluatiuns were pcrformed during this reporting period. 
3.2.6 Electric and Hybrid Vehicle $R \& D$

3.2.6.1 EHV Program Coordination Support (JO 7765)

Objective: To provide close support to the EHV $R$ \& $D$ Coordinating Committee in the areas of analysis, assessment, and planning.

Status: The following activities were in progress during the reporting period, or are of a continuing nature.

1. General Support to the EHV Coordinating Committee

Objective: To participate in bimonthly $R$ \& $D$ work sessions and provide planning support.

Status: Aerospace participated in the Quarterly Project Review of the EHV activities at the four national laboratories.

2. Preparation of Level II R \& D Plan for EHV Program

Objective: To expand the current Level I Program Plan to a more definitive and descriptive version (Level II).

Status: The Vehicle Systems timeline chart has been revised to reflect the new program organization. Inputs to the project descriptions are still being received from DOE Headquarters personnel. Work on the plan itself has had to slip due to higher priority tasks. A May.delivery of the plan to the General Accounting Office is now scheduled.

3. Goals Study

Objective: The major thrust of this activity is the formulation of goals for the EHV R \& D Program based on the Level I and Level II PIans.

Status: No new activity in this area.

4. Miscellaneous Support Activities

Objective: To perform various quick-response support tasks as requested by the $R$ \& $D$ Coordinating Committee.

Status: At the request of DOE, Aerospace is in the process of contracting with firms to assist in technical information dissemination for the $R \& D$ Program. It is anticipated that these firms will be on subcontract by mid-April. 


\subsubsection{Electric and Hybrid Vehicle Analysis Support (JO 7766)}

Objective: This task area encompasses assignments of analysis and assessment of electric and hybrid vehicle systems and components.

Status: The following activities were in progress during the reporting period.

1. Proposal/Report/Correspondence Analysis

Objective: This effort includes detailed analyses of proposals and reports, reviews of the DOE EHV Annual and Quarterly Reports, and reviews of miscellaneous DOE reports, as well as preparation of technical responses to inquiries.

Status: No activity during this period.

2. Development of Technique for Including Electric Vehicles in Corporate Average Fuel Economy (CAFE) Standards

Objective: To develop an approach and technique whereby the energy-useequivalence of electric vehicles could be incorporated into the CAFE standards.

Status: This area has been the primary focus during the reporting period. The CAFE briefing has been presented to Garrett/AiResearch (March 15), Jet Propulsion Laboratory (March 16), The Aerospace Corporation/Mobile Systems Group (March 19), and General Motors (March 27). These briefings have been very beneficial. Numerous excellent comments and offers of assistance were received. The meeting with General Motors was particularly valuable. Based on these comments, several revisions and expansions of the briefing have been made.

A meeting with Chrysler personnel has been scheduled for April 25. No fixed date has been set as yet for Ford. With Senator McClure's introduction of Senate Bill 624, interest in the subject has greatly increased among the auto manufacturers. It is hoped that these industry briefings can be completed in April.

\subsubsection{Electric and Hybrid Vehicle Performance Standards Support (JO 7767)}

Objective: This task involves analyses of the current state of the art of the performance capabilities of EHV technology to provide a basis for recommending 
future-year EHV performance standards. It will provide DOE with a defensible basis for setting future standards. The first cycle is to be completed by December 1979 .

Status: During this reporting period, discussions continued with individuals representing interested organizations who have comments on the existing demonstration performance standards. During this month, 15 companies or organizations were contacted. Interview material has been written for the rough draft of the report which will go to DOE. A list of potential recommended performance standards changes has been drafted.

4.

\section{CURRENT STATUS OF ALL ACTIVITIES}

Appendix $A$ and Section 3 above describe the specific assignments made to date and their general schedule requirements. Figure 1 depicts this information in graphical format. As can be noted, much of the effort is of the continuing, quick-response, or intermittent nature.

Appendix B is a summary table which delineates the principal reporting activities of The Aerospace Corporation on all task assignments from the inception of the contract to date.

5.

PROBLEM AREAS

No major problems of a technical nature were encountered during this reporting period.

6.

PLANNED ACTIVITIES FOR APRIL 1979

The month of April will be devoted to continuing those task activities under way, and in initiating any newly-defined tasks thal may be assigned during that period.

7 .

EXPENDITURE STATUS

The currently obligated funding level is $\$ 1,060,000$, with an additional $\$ 940,000$ currently being processed for a total funding level to date of $\$ 2,000,000$. The apportionment of these funds by Job Order (JO) is summarized in Table 2, together with an accounting of cumulative expenditures on a monthly basis.

The cumulative actual total expenditure through this reporting period is $\$ 758,000$. 


\section{Table 1. JO's Assigned}

Heat Engine Systems

7751

Automotive Technology Assessment Study

7752

Technical Responses to Inquiries

7753

Highway Systems Analysis Support

7754

Evaluation of Specific Engines/Systems

7755

Special Heat Engine System Studies

Alternative Fuels

7756 Distillery Impact Study

7757 Immediate Action Studies Support

7758 Fuel Economy Improvement Potential Study

7759 Alternative Fuels Analysis Support

$7760 \quad$ Special Alternative Fuels Studies

Nonhighway Systems

7761 Commercial Aviation Fuel Economy Study

7762 General Aviation Fuel Economy Study

7763 Nonhighway Systems Analysis Support

New Concepts Evaluation

7764. Proposal Evaluations

Electric and Hybrid Vehicles

7765 . EHV Program Coordination Support

7766 Electric and Hybrid Vehicle Analysis Support

7767 Electric and Hybrid Vehicle Performance Standards Support

Technology Assessment and Implementation

7768 Assessment and Implementation Overview

Technical Program Documentation

7769 Program Planning and Analysis

7770 Management Review and Control Documentation 
Table 2. Cumulative Expenditure Fistory with Job Order Breakdown

\begin{tabular}{|c|c|c|c|c|c|c|c|c|c|c|c|c|}
\hline $\begin{array}{l}\text { Job Order } \\
\text { Number }\end{array}$ & Task Description & $\begin{array}{l}\text { Fresently } \\
\text { Allocated } \\
\text { Funds(1) }\end{array}$ & $\begin{array}{c}\text { Actual } \\
\text { Expenditure } \\
\text { Through } \\
7 / 31 / 78\end{array}$ & $\begin{array}{c}\text { Actual } \\
\text { Expenditure } \\
\text { Through } \\
8 / 31 / 78\end{array}$ & \begin{tabular}{|} 
Actual \\
Expenditure \\
Through \\
$9 / 30 / 78$
\end{tabular} & $\begin{array}{c}\text { Actual } \\
\text { Expenditure } \\
\text { Through } \\
10 / 31 / 78\end{array}$ & $\begin{array}{l}\text { Actuit } \\
\text { (ixpenditure } \\
\text { Through } \\
11 / 36 / 78\end{array}$ & $\begin{array}{l}\text { Actual } \\
\text { Pxpendi ture } \\
\text { Through } \\
12 / 31 / 78\end{array}$ & $\begin{array}{c}\text { Actuil } \\
\text { Expenditure } \\
\text { Through } \\
1 / 31 / 79\end{array}$ & \begin{tabular}{|} 
Actual \\
Expcondi ture \\
Through \\
$2 / 28 / 79$
\end{tabular} & $\begin{array}{l}\text { Actual } \\
\text { Exponditure } \\
\text { Through } \\
3 / 31 / 79\end{array}$ & \\
\hline $\begin{array}{l}7751 \\
7752 \\
7753 \\
7754 \\
7755\end{array}$ & $\begin{array}{l}\text { HEAT ENGINE SYSTEMS } \\
\text { Automotive Technology Assessment } \\
\text { Technical Responses to Inquiries } \\
\text { Highway Systems Analysis Support } \\
\text { Evaluation of Specific Engines/Systems } \\
\text { Special Heat Engine System Studies }\end{array}$ & $\$ 9$ 9uk & $\begin{array}{l}(34,900) \\
2,100 \\
12,700 \\
20,100 \\
0 \\
0\end{array}$ & $\begin{array}{c}(98,600) \\
3,000 \\
19,500 \\
76,100 \\
0 \\
0\end{array}$ & $\begin{array}{c}(143,900) \\
17,900 \\
24,300 \\
97,400 \\
4,300 \\
0\end{array}$ & $\begin{array}{c}(173,500) \\
29,400 \\
28,500 \\
106,000 \\
9,600 \\
0\end{array}$ & $\begin{array}{c}(2.95,000) \\
33,900 \\
34,600 \\
122,500 \\
14,000 \\
0\end{array}$ & $\begin{array}{c}(243,700) \\
\quad 15,100 \\
41,200 \\
143,400 \\
14,000 \\
0\end{array}$ & $\begin{array}{l}(287,800) \\
50,200 \\
45,700 \\
177,900 \\
14,000 \\
0\end{array}$ & $\begin{array}{c}(324,100) \\
56,600 \\
53,100 \\
199,800 \\
14,600 \\
0\end{array}$ & $\begin{array}{c}(371,700) \\
57,400 \\
63,000 \\
236,700 \\
14,600 \\
0\end{array}$ & \\
\hline $\begin{array}{l}7756 \\
7757 \\
7758 \\
7759 \\
7760 \\
\end{array}$ & $\begin{array}{l}\text { ALTERNATIVE FUELS } \\
\text { Distillery Inpact Stuly } \\
\text { In:mediate Action Studies Support } \\
\text { Fcel Economy Improzement Potential } \\
\text { Alte rnative Fuels Analysis Support } \\
\text { Special Alternative Fuels Studies } \\
\end{array}$ & $\$ 250 \mathrm{~K}$ & $\begin{array}{c}(19,400) \\
5,200 \\
0 \\
1,700 \\
12,500 \\
0 \\
\end{array}$ & $\begin{array}{c}(47,200) \\
8,200 \\
9,600 \\
6,000 \\
23,400 \\
0\end{array}$ & $\begin{array}{c}(62,600) \\
9,200 \\
15,100 \\
10,700 \\
27,600 \\
0 \\
\end{array}$ & $\begin{array}{c}(71,200) \\
9,400 \\
17,900 \\
10,700 \\
33,200 \\
0 \\
\end{array}$ & $\begin{array}{c}(79,900) \\
11,700 \\
18,000 \\
12,300 \\
37,500 \\
400 \\
\end{array}$ & $\begin{array}{r}(105,600) \\
15,200 \\
18,100 \\
17,900 \\
54,000 \\
400 \\
\end{array}$ & $\begin{array}{c}(126,000) \\
15,200 \\
18,100 \\
23,600 \\
68,600 \\
500 \\
\end{array}$ & $\begin{array}{r}(145,500) \\
16,500 \\
18,300 \\
29,900 \\
80,300 \\
500 \\
\end{array}$ & $\begin{array}{c}(175,900) \\
19,300 \\
18,800 \\
36,800 \\
100,50 \\
56 \\
\end{array}$ & \\
\hline $\begin{array}{l}7761 \\
7762 \\
7763 \\
\end{array}$ & $\begin{array}{l}\text { NONHIGHWAY SYSTEMS } \\
\text { Conmercial Aviatior. Fuel Economy } \\
\text { General Aviation Fuel Econony } \\
\text { Nonhighway Systems Analys is Support }\end{array}$ & $\$ 220 \mathrm{~K}$ & $\begin{array}{l}(10,500) \\
10,500 \\
0 \\
0 \\
\end{array}$ & $\begin{array}{c}(13,100) \\
13,100 \\
0 \\
0 \\
\end{array}$ & $\begin{array}{c}(17,300 \\
16,200 \\
0 \\
1,100 \\
\end{array}$ & $\begin{array}{c}(18,300) \\
16,900 \\
0 \\
1,400 \\
\end{array}$ & $\begin{array}{c}(21,000) \\
19,600 \\
0 \\
1,400 \\
\end{array}$ & $\begin{array}{c}(29,800) \\
28,400 \\
0 \\
1,400 \\
\end{array}$ & $\begin{array}{c}(37,900\} \\
36,500 \\
0 \\
1,400 \\
\end{array}$ & $\begin{array}{c}(45,900) \\
44,500 \\
0 \\
1,400 \\
\end{array}$ & $\begin{array}{c}(55,800) \\
54,400 \\
0 \\
1,400 \\
\end{array}$ & \\
\hline 7764 & $\begin{array}{l}\text { NEW CONCEPTS EVALUATION } \\
\text { PEoposal Evaluations } \\
\end{array}$ & $\$ 60 \mathrm{~K}$ & $\begin{array}{r}(2,100) \\
2,100 \\
\end{array}$ & $\begin{array}{l}(2,900) \\
2,900 \\
\end{array}$ & $\begin{array}{c}(4,700) \\
4,700\end{array}$ & $\begin{array}{r}(5,100) \\
5,100 \\
\end{array}$ & $\begin{array}{c}(8,800) \\
8,800 \\
\end{array}$ & $\begin{array}{c}(13,100) \\
13,100\end{array}$ & $\begin{array}{c}(13,100) \\
13,100\end{array}$ & $\begin{array}{c}(14,200) \\
14,200)\end{array}$ & $\begin{array}{c}(15,100) \\
15,100 \\
\end{array}$ & \\
\hline $\begin{array}{l}7: 65 \\
7: 66 \\
7 \div 67\end{array}$ & $\begin{array}{l}\text { EIECTRIC \& HYBRID VEHICLE R \& D } \\
\text { EHV Program Coordination Support } \\
\text { E.ectric Vehicle Analysis Support } \\
\text { H.jbrid Vehicle Analysis Support }\end{array}$ & $\$ 570 K$ & $\begin{array}{l}(10,300) \\
10,300 \\
0 \\
0\end{array}$ & $\begin{array}{c}(24,500) \\
24,500 \\
0 \\
0 \\
\end{array}$ & $\begin{array}{c}(46,200 \\
46,200 \\
0 \\
0\end{array}$ & $\begin{array}{c}(57,900) \\
57,900 \\
0 \\
0\end{array}$ & $\begin{array}{c}(72,500) \\
72,300 \\
200 \\
0\end{array}$ & $\begin{array}{r}(87,300) \\
83,300 \\
3,500 \\
500 \\
\end{array}$ & $\begin{array}{c}(100,300) \\
92,600 \\
5,900 \\
1,800 \\
\end{array}$ & $\begin{array}{c}(118,700) \\
101,500 \\
11,000 \\
6,200 \\
\end{array}$ & $\begin{array}{c}(139,400) \\
113,300 \\
14,000 \\
12,100 \\
\end{array}$ & \\
\hline $7 ? 68$ & $\begin{array}{l}\text { TECHNOLOGY ASSESSMENT } \\
\text { Assessinent and Implemeritation Ove rview }\end{array}$ & $\$ 0$ & $\begin{array}{lll}1 & 0 & 1 \\
0 & 0\end{array}$ & $\begin{array}{c}(0) \\
0 \\
\end{array}$ & $\left(\begin{array}{l}0 \\
0\end{array}\right)$ & $\left(\begin{array}{l}0 \\
0 \\
0\end{array}\right.$ & $\left(\begin{array}{l}0 \\
0\end{array}\right)$ & $\begin{array}{r}\left(\begin{array}{l}0 \\
0\end{array}\right) \\
0\end{array}$ & $\left(\begin{array}{l}0 \\
0\end{array}\right)$ & $\begin{array}{l}\left(\begin{array}{l}0 \\
0\end{array}\right) \\
0\end{array}$ & $\left(\begin{array}{l}0 \\
0\end{array}\right)$ & \\
\hline \multirow[t]{2}{*}{$\begin{array}{l}7769 \\
7770\end{array}$} & $\begin{array}{l}\text { TECHNIC L PROGR.AM DOCUMENTATION } \\
\text { Program Planning a.2d Analysis } \\
\text { Managenient Revicw and Control Document }\end{array}$ & * & $\begin{array}{l}\left(\begin{array}{l}1 \\
0 \\
0 \\
0\end{array}\right. \\
0\end{array}$ & $\begin{array}{l}\left(\begin{array}{l}0 \\
0\end{array}\right) \\
0 \\
0\end{array}$ & $\begin{array}{l}\left(\begin{array}{l}0 \\
0\end{array}\right) \\
0 \\
0\end{array}$ & $\begin{array}{l}\left(\begin{array}{l}0 \\
0\end{array}\right) \\
0 \\
0 \\
\end{array}$ & $\begin{array}{l}(100) \\
100 \\
0\end{array}$ & $\begin{array}{l}(100) \\
100 \\
0\end{array}$ & $\begin{array}{l}(100) \\
100 \\
0\end{array}$ & $\begin{array}{l}(100) \\
0^{100} \\
\end{array}$ & $\begin{array}{l}(100) \\
100 \\
0\end{array}$ & \\
\hline & TOTALS & $\$ 2,000$ & 77,200 & 186,300 & 274,700 & 326,000 & $3.37,300$ & 479,600 & 565,200 & $6,18,500$ & 758,000 & \\
\hline
\end{tabular}

"Assignnients to be charged pro-rata to other funding categories.

(1) Inclules $\$ 940,060$ being processed as of $4 / 6 / 79$ 


\begin{tabular}{|c|c|c|c|c|c|c|c|c|c|c|}
\hline $\begin{array}{l}\text { Job } \\
\text { Order } \\
\text { Namber } \\
\end{array}$ & Task Description & $\begin{array}{c}\text { July } \\
1978 \\
\text { Expenditure }\end{array}$ & $\begin{array}{c}\text { August } \\
1978 \\
\text { Expenditure } \\
\end{array}$ & $\begin{array}{c}\text { September } \\
1978 \\
\text { Expenditure }\end{array}$ & \begin{tabular}{c|} 
October \\
1978 \\
Expenditure \\
\end{tabular} & $\begin{array}{l}\text { November } \\
1978 \\
\text { Expenditure }\end{array}$ & $\begin{array}{c}\text { Deceinber } \\
1978 \\
\text { Expenili ture }\end{array}$ & $\begin{array}{c}\text { January } \\
1979 \\
\text { Expendi ture }\end{array}$ & $\begin{array}{c}\text { liebruary } \\
1979 \\
\text { lixpendi ture }\end{array}$ & $\begin{array}{l}\text { Mapin } \\
19 \\
\text { Lipel.....ne }\end{array}$ \\
\hline & HEAT ENGINE SYSTEMS & $(34,900)$ & $(63,700)$ & $(45,300)$ & $(29,600)$ & $(31,500)$ & $(38.700)$ & $(44,100)$ & $(36,300)$ & $(47,600)$ \\
\hline $\begin{array}{l}7751 \\
7752 \\
7753 \\
7754 \\
7755\end{array}$ & $\begin{array}{l}\text { Automotive Technology Assessment } \\
\text { Technical Responses to Inquiries } \\
\text { Highway Systems Analysis Support } \\
\text { Evaluation of Specific Engines/Systems } \\
\text { Special Heat Engine System Studies }\end{array}$ & $\begin{array}{l}2,100 \\
12,700 \\
20,100 \\
0 \\
0\end{array}$ & $\begin{array}{l}900 \\
6,800 \\
56,000 \\
0 \\
0\end{array}$ & $\begin{array}{r}14,900 \\
4,800 \\
21,300 \\
4,300 \\
0\end{array}$ & $\begin{array}{l}11,500 \\
4,200 \\
8,600 \\
5,300 \\
0\end{array}$ & $\begin{array}{l}4,500 \\
6,100 \\
16,500 \\
4,400 \\
0\end{array}$ & $\begin{array}{l}11,200 \\
6,600 \\
20,900 \\
0 \\
0\end{array}$ & $\begin{array}{l}5,100 \\
4,500 \\
34,500 \\
0 \\
0\end{array}$ & $\begin{array}{r}6,400 \\
7,400 \\
21,900 \\
600 \\
0\end{array}$ & $\begin{array}{l}800 \\
9,900 \\
36,900 \\
0 \\
0\end{array}$ \\
\hline $\begin{array}{l}7756 \\
7757 \\
7758 \\
7759 \\
7760\end{array}$ & $\begin{array}{l}\text { A LTERNATIVE FUELS } \\
\text { Distillery Impact Study } \\
\text { Immediate Action Studies Support } \\
\text { Fuel Economy Improvement Potential } \\
\text { Alternative Fuels Analysis Support } \\
\text { Special Alternative Fuels Studies }\end{array}$ & $\begin{array}{c}(19,400) \\
5,200 \\
0 \\
1,700 \\
12,500 \\
0\end{array}$ & $\begin{array}{l}(27,800) \\
3,000 \\
9,600 \\
4,300 \\
10,900 \\
0\end{array}$ & $\begin{array}{l}(15,400) \\
1,000 \\
5,500 \\
4,700 \\
4,200 \\
0\end{array}$ & $\begin{array}{l}(8,600) \\
200 \\
2,800 \\
0 \\
5,600 \\
0\end{array}$ & $\begin{array}{r}(8,700) \\
2,300 \\
100 \\
1,600 \\
4,300 \\
400\end{array}$ & $\begin{array}{l}(25,700) \\
3,500 \\
100 \\
5,600 \\
16,500 \\
0\end{array}$ & $\begin{array}{c}(20,400) \\
0 \\
0 \\
5,700 \\
14,600 \\
100\end{array}$ & $\begin{array}{c}(19,500) \\
1,300 \\
200 \\
6,300 \\
11,700 \\
0\end{array}$ & $\begin{array}{c}(30,400) \\
2,800 \\
500 \\
6,900 \\
20,200 \\
0\end{array}$ \\
\hline $\begin{array}{l}7761 \\
7762 \\
7763\end{array}$ & $\begin{array}{l}\text { NONHIGHWAY SYSTEMS } \\
\text { Commercial Aviation Fuel Economy } \\
\text { General Aviation Fuel Economy } \\
\text { Nonhighway Systems Analys is Support }\end{array}$ & $\begin{array}{l}(10,500) \\
10,500 \\
0 \\
0\end{array}$ & $\begin{array}{l}(2,600) \\
2,600 \\
0 \\
0\end{array}$ & $\begin{array}{c}(4,200) \\
3,100 \\
0 \\
1,100\end{array}$ & $\begin{array}{c}(1,000) \\
700 \\
0 \\
300\end{array}$ & $\begin{array}{l}(2,700) \\
2,700 \\
0 \\
0\end{array}$ & $\begin{array}{l}(8,800) \\
8,800 \\
0 \\
\dot{0}\end{array}$ & $\begin{array}{l}(8,100) \\
8,100 \\
0 \\
0\end{array}$ & $\begin{array}{l}(8,0(10)) \\
8,000 \\
0 \\
0\end{array}$ & $\begin{array}{l}(9,900) \\
9,900 \\
0 \\
0\end{array}$ \\
\hline 7764 & $\begin{array}{l}\text { NEW CONCEPTS EVALUATION } \\
\text { Proposal Evaluations }\end{array}$ & $\begin{array}{c}(2,100) \\
2,100 \\
\end{array}$ & $\begin{array}{c}(800) \\
800\end{array}$ & $\begin{array}{c}(1,800) \\
1,800\end{array}$ & $\begin{array}{c}(400) \\
400\end{array}$ & $\begin{array}{c}(3,700) \\
3,700\end{array}$ & $\begin{array}{c}(4,300) \\
4,300 \\
\end{array}$ & $\begin{array}{l}\left(\begin{array}{ll}0 & \end{array}\right) \\
\left(\begin{array}{ll}0 & )\end{array}\right)\end{array}$ & $\begin{array}{c}(1,100) \\
1,100 \\
\end{array}$ & 100 \\
\hline $\begin{array}{l}7765 \\
7766 \\
7767 \\
\end{array}$ & $\begin{array}{l}\text { ELECTRIC GND HYBRID VEHICLE R \& D } \\
\text { EHV Program Coordination Support } \\
\text { Electric Vehicle Analysis Support } \\
\text { Hybrid Vehicle Analysis Support } \\
\end{array}$ & $\begin{array}{c}(10,300) \\
10,300 \\
0 \\
0\end{array}$ & $\begin{array}{l}(14,200) \\
14,200 \\
0 \\
0\end{array}$ & $\begin{array}{c}(21,700) \\
21,700 \\
0 \\
0 \\
\end{array}$ & $\begin{array}{l}(11,700) \\
11,700 \\
0 \\
0 \\
\end{array}$ & $\begin{array}{l}(14,600) \\
14,400 \\
200 \\
0\end{array}$ & $\begin{array}{r}(14,800) \\
11,000 \\
3,300 \\
500\end{array}$ & $\begin{array}{r}(13,000) \\
9,300 \\
2,400 \\
1,300 \\
\end{array}$ & $\begin{array}{r}(18,400) \\
8,900 \\
5,100 \\
4,400 \\
\end{array}$ & $\begin{array}{c}(20,700) \\
11,800 \\
3,000 \\
5,900\end{array}$ \\
\hline 7768 & $\begin{array}{l}\text { TECHNOLOGY ASSESSMENT } \\
\text { Assessment and Implementation Overview }\end{array}$ & $\left.\begin{array}{c}0 \\
0 \\
0\end{array}\right)$ & $\begin{array}{c}10 \\
0\end{array}$ & $\left(\begin{array}{l}0 \\
0\end{array}\right)$ & $\left(\begin{array}{l}0 \\
0\end{array}\right)$ & $\left(\begin{array}{l}0 \\
0\end{array}\right)$ & $\left(\begin{array}{l}0 \\
0\end{array}\right)$ & $\left(\begin{array}{l}0 \\
0\end{array}\right)$ & $\left(\begin{array}{l}0 \\
0\end{array}\right)$ & $\begin{array}{r}(0) \\
0\end{array}$ \\
\hline \multirow[t]{2}{*}{$\begin{array}{l}7769 \\
7770\end{array}$} & $\begin{array}{l}\text { TECHNICAL PROGRAM DOCUMENTATION } \\
\text { Program Planning and Analysis } \\
\text { Management Review and Control Document }\end{array}$ & $\begin{array}{l}(0) \\
0 \\
0\end{array}$ & $\begin{array}{l}(0) \\
0 \\
0\end{array}$ & $\left(\begin{array}{ll}0 & 1 \\
0 \\
0\end{array}\right)$ & $\begin{array}{l}\left(\begin{array}{l}0 \\
0\end{array}\right) \\
0 \\
0\end{array}$ & $0^{(100)}$ & $\left(\begin{array}{l}0 \\
0 \\
0 \\
1\end{array}\right)$ & 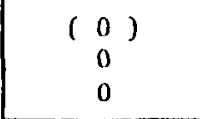 & $\left(\begin{array}{l}0 \\
0 \\
0\end{array}\right)$ & $\left(\begin{array}{l}0 \\
0 \\
0\end{array}\right)$ \\
\hline & TOTALS & 77,200 & 109,100 & 88,400 & 51,300 & 61,300 & 92,300 & 85,600 & 83,300 & 109,500 \\
\hline
\end{tabular}


Figure 1. Program Schedule

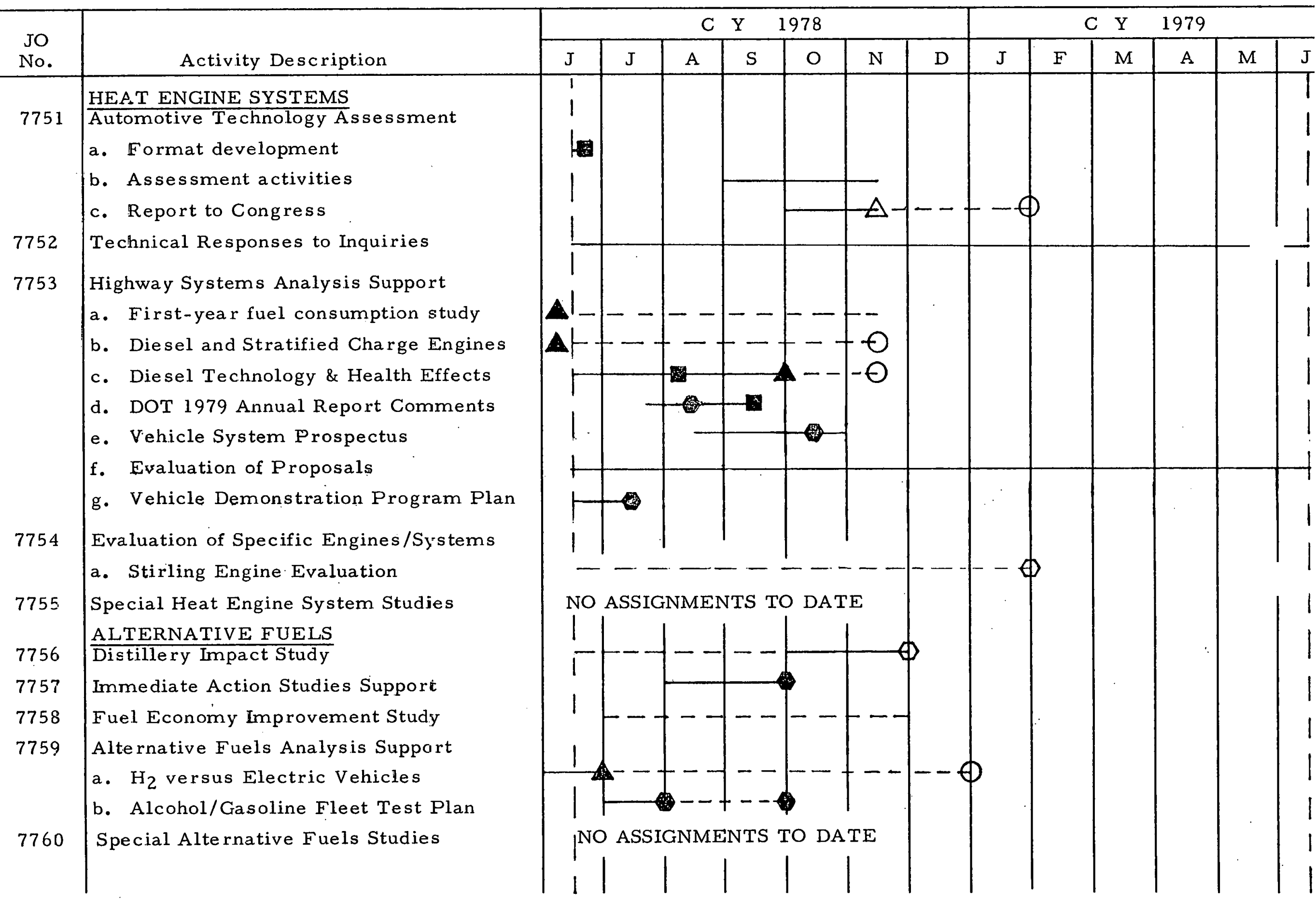


Figure 1. Program Schedule (continued)

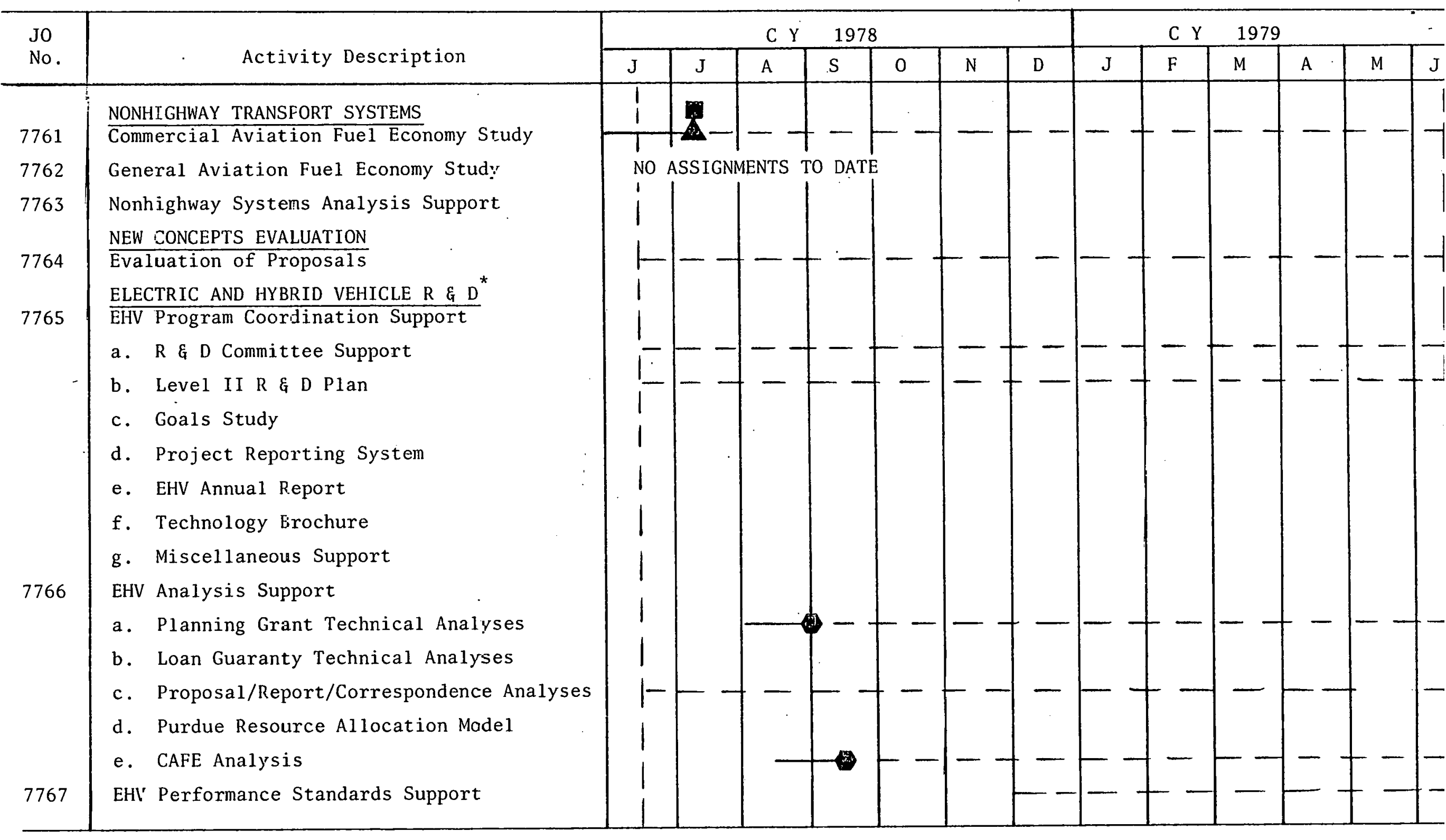

${ }^{*}$ Revised 12/10/78 
Figure 1. Program Schedule (continued)

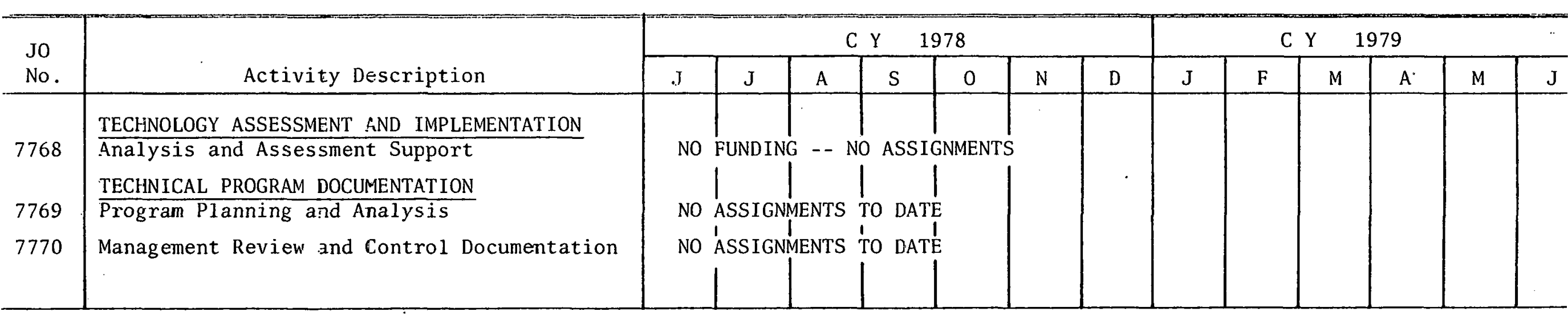

\section{LEGEND}

Activity of a continuous nature

Activity of a low-level or intermittent nature

Formal final task report

$\triangle \quad$ Rough draft of task report

0 Informal draft or working memorandum for DOE/TEC internal use

口 Briefing

Open symbols represent target or planned milestones; closed symbols represent completed milestones or activities. 
Figure 2. Cumulative Expenditure History

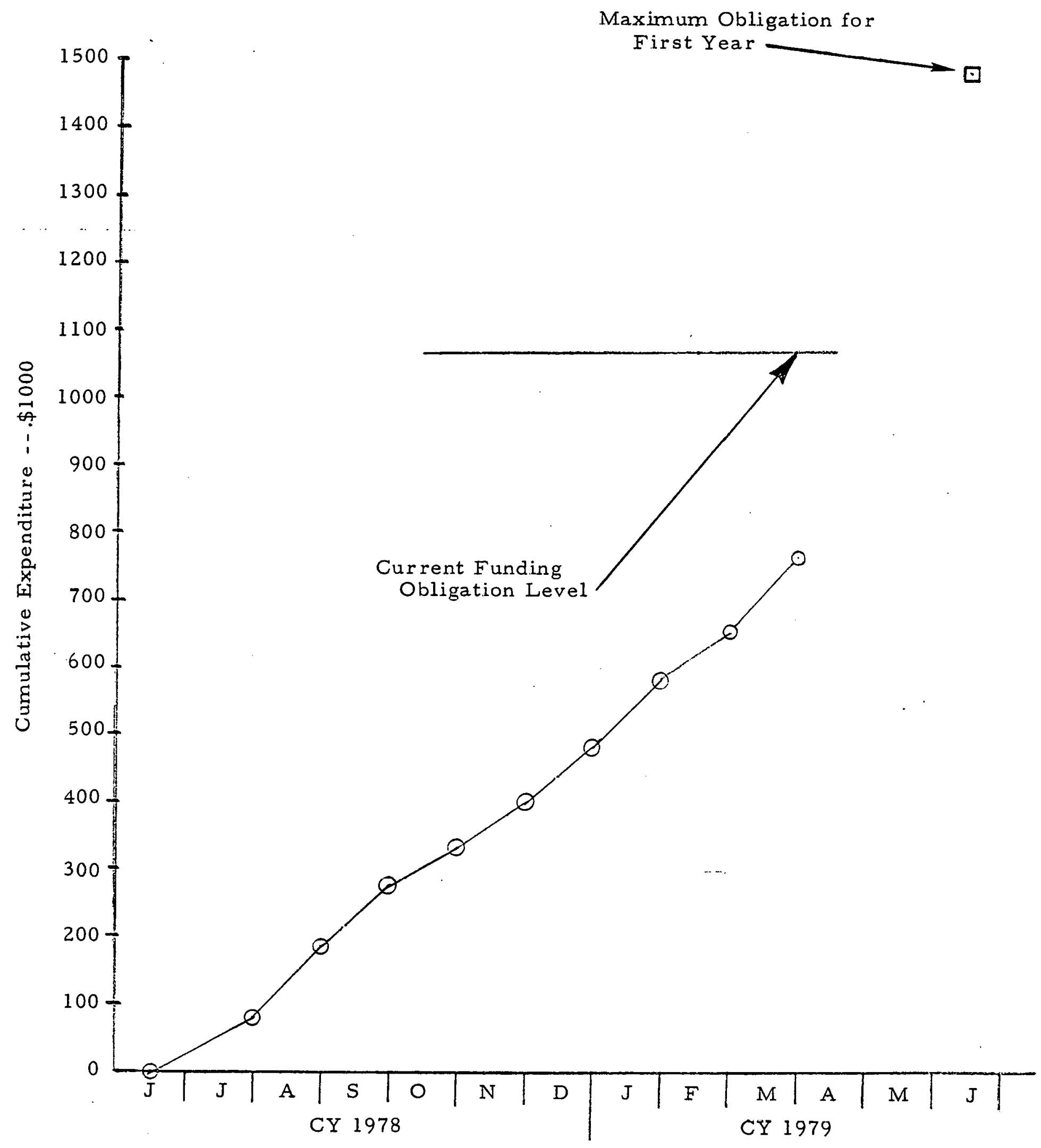


- APPENDIX A

\section{SUMMARY OF TASK ASSIGNMENTS}

1. General Scope of Effort

The work to be performed by The Aerospace Corporation for the DOE/TEC is confined to the following basic task areas.

Task 1 - Technical Support of Ongoing Research and Development Programs in Energy Efficient Transportation Systems

Task 2 - Analysis for the Future Commercialization of Transportation Technologies

Task 3 - New Concept Evaluation Program Support

Task 4 - Technical Evaluation of New Concepts, Inventions, and Ideas

Task 5 - Assessment of Technological and Other Factors on the Implementation and Utilization of Transportation in the United States

Task 6 - Program Planning Analysis and Documentation

Within each of these basic areas, Aerospace may be called upon to perform analyses, assessments, evaluations, plans, reports, etc., as required to support DOE/TEC programs.

2. Specific Assignments to Date and Schedule Requirements

The following assignments have been in effect or initiated since the inception of work on June 15, 1978.

$2.1 \quad$ Heat Engine Systems

2.1.1 Automotive Technology Assessment Study (JO 7751)

Section 310 of the Automotive Propulsion Research and Development Act of 1978 (Title III of Public Law 95-238) requires that DOE/TEC submit an annual report to Congress on the activities DOE/TEC has performed in implementing the provisions of the Act. The effort in this task is to assist DOE/TEC in preparing this annual report, by means of technology assessments, 
planning, and report writing and coordination. A rough draft of the report is to be completed by November 30, 1978; the final report is to be published by February 1979 .

\subsubsection{Technical Responses to Inquiries (J0 7752)}

DOE/TEC receives many inquiries and suggestions concerning transportation energy conservation from inventors, Congressional offices, and concerned citizens. The effort in this task is to provide quick-response support to DOE/TEC by reviewing the technical content of such letters of inquiry and preparing technical inputs for letter responses. This activity is expected to be of a continuing nature throughout the contract period.

\subsubsection{Highway Systems Analysis Support (JO 775.3)}

As a consequence of program changes or developments in DOE/TEC or industry $R$ \& $D$ status, DOE/TEC is required to investigate, evaluate, and report on diverse technological issues or topics. The effort in this task is to provide technical analysis and assessment support to DOE/TEC in completing these many and varied requirements. The time involved varies, depending upon the specific area of investigation, and may be as short as a few weeks or as long as six months.

Task assignments to date include:

1. Fuel Consumption and Emission Characteristics of Light-duty Diesel and Stratified. Charge Engines

2. First-year Fuel Consumption of New Automobiles, Light-duty Trucks, Heavy-duty Trucks, Buses, and Motorcycles

3. Summary of viesèl-related Wurk in Areas of Technology Development and Health Effects

4. DOE/TEC Technical Contributions to DOT's 1979 Annual Report to Congress

5. Preparation of Prospectus of Requirements for Vehicle Systems Program

6. Evaluation of Numerous Unsolicited Proposals

7. Development of Demonstration Program Plan for Gas Turbine and Stirling-engine-powered Automobiles 
Items 1 and 2 above were initiated during the previous contract and are being continued and completed during the present contract.

\subsubsection{Evaluation of Specific Engines/Systems (JO 7754)}

This $J O$ is reserved for prospective assignments of evaluations of specific engines and/or vehicle systems which are deemed to require an extensive level of effort and/or require a separate accounting of expended effort. One assignment in this category has been made to date.

1. Evaluation of Stirling-engine-powered Vehicle Provided by United Stirling of Sweden

\subsubsection{Special Heat Engine System Studies (JO 7755)}

This JO is reserved for prospective assignments of longer-range study activities which are expected, at the outset, to result in formal publications of The Aerospace Corporation. No assignments have yet been made in this category.

\section{$2.2 \quad$ Alternative Fuels Utilization \\ 2.2.1 Distillery Impact Study (JO 7756)}

The DOE Alcohol Fuels Program Plan (Document No. DOE/US-0001/2, March 1978) establishes DOE's role and plans for establishing alcohol fuel availability and end-use commercialization potential. The effort in this task is to determine the role that the distilling industry can fill in producing alcohol for fuel use. The study is examining the technological, economic, institutional, and environmental characteristics of the fermentation industries producing industrial ethanol and distilled spirits. The study is scheduled for completion in October 1978.

\subsubsection{Immediate Action Studies Support (JO 7757)}

The DOE Alcohol Fuels Program Plan incorporates the requirement for a series of studies to examine and help resolve key technological, economic, environmental, and institutional issues that obstruct or cloud commercialization decisions. As a consequence, an Alcohol Fuels Policy Review by DOE has been instituted. The effort in this task is to provide analysis 
support to the currently-operative Alcohol Fuels End-use Task Force and to any subsequent immediate-action studies in this area. The current effort for the End-use Task Force is expected to end by 30 September 1978 .

2.2.3 Fuel Economy Improvement Potential Study (J0 7758)

The effort in this two-phase study is to determine the combustion efficiency potential of spark ignition engines and the potential impact of fuel modifications. Phase I involves the analysis of the combustion losses of several late model engines, and is expected to be completed by the end of September 1978. Phase II involves a theoretical analysis of combustion inefficiencies as affected by variations in fuel heat release rate. A completion date for Phase II has not yet been determined.

2.2.4 Alternative Fuels Analysis Support (JO 7759)

The effort in this task is directed to providing analysis and assessment support in response to changing program needs or the requirement to respond to technological questions raised by developments in DOE and industry programs. The time involved varies, depending upon the specific area of investigation, and is expected to range from a few weeks to several months.

Task assignments to date include:

1. Assessment of $\mathrm{H}_{2}$ vs. Battery-powered Automobiles (this is a continuation of study initiated under previous contract)

2. Preparation of Project Plan for Reliability Fleet Testing of Alcohol/Gasoline Blends

2.2.5 Special Alternative Fue1s Studies (JO 7760)

This. JO is reserved for prospective assignments of longer-range study activities which are expected, at the outset, to result in formal publications of The Aerospace Corporation. No assignments have yet been made in this category.

$2.3 \quad$ Nonhighway Transport Systems

2.3.1 Commercial Aviation Fuel Economy Study (JO 7761)

The effort in this study task is to examine commercial aviation operational policies and fuel conservation strategies and identify those 
that are the most beneficial and deserving of DOE support. The basic study effort and report rough draft were completed under the previous contract. Effort is currently under way to define the next level of study activity required and to define a meaningful schedule for accomplishing the work.

\subsubsection{General Aviation Fuel Economy Study (j0 7762)}

This JO is reserved for prospective assignment of a study which would examine the operational policies and fuel conservation strategies which would be most beneficial to the category of general aviation. It would thus parallel the Commercial Aviation Fuel Economy Study noted above in Section 2.3.1. No assignment has yet been made in this category.

2.3.3 Nonhighway Systems Analysis Support (JO 7763)

This JO is reserved for prospective assignments of analysis and assessment support in the air, rail, pipeline, and marine transport sectors, as required. One assignment has been made to date.

1. Optimization of Rail Car Structures for Energy Conservation (analysis and assessment of evaluation criteria)

$2.4 \quad$ New Concepts Evaluation (JO 7764)

The effort in this task is (1) to conduct technical evaluations of new concepts, inventions, and ideas offered to DOE/TEC by industry, universities, government, and individuals, and (2) to conduct feasibility studies for the implementation and effective utilization of developments arising from the New Concepts Program of TEC. This activity is of a continuing nature and is expected to continue throughout the contract period. $2.5 \quad$ Electric and Hybrid Vehicle R \& D

On November 6,1978 , the EHV $R$ \& D support tasks were restructured as defined below in Sections 2.5.1 through 2.5.3. Refer to the Third Monthly Progress Report of October 1978 (7769.78.MGH-229) for assignments prior to November 6, 1978.

2.5.1 EHV Program Coordination Support (JO 7765)

In accordance with the provisions of the Electric and Hybrid Vchiclc (EHV) Research, Development and Demonstration Act of 1976, DOE/TEC 
has instituted an $R \& D$ program which will culminate in the demonstration of up to 10,000 vehicles in in-use service.

DOE/TEC has established an EHV R \& D Coordinating Committee to facilitate coordination and management of the various participants in the $R$ \& $D$ program. The effort in this task is to provide close support to that Committee in the areas of analysis, assessment, and planning. This activity is of a continuing nature and is expected to continue throughout the contract period. Specific task assignments as of November 6, 1978 include:

1. $R \& D$ Committee Support

This task is a continuation and expansion of the previous assignment in this area. The expanded effort consists of careful review of laboratory and contractor monthly reports to advise the Committee of potential problem areas.

2. Level II $R$ \& D Plan

This is anticipated to be a major effort during the first half of FY 79 .

3. Goals Study

This new task is to be a principal study during the year. Its thrust is the formulation of goals for the EHV R \& D Program based on the Level I and Level II Plans.

4. Project. Reporting System

This new task entails working with HQ staff to devise a project reporting system to allow HQ management closer cognizance and control over the field projects.

5. EHV Annual Report

This new task involves the preparation of the $R$ \& $D$ Program chapter in the next EHV Annual Report.

6. Technology Brochure Preparation

This task involves assistance in the preparation of a brochure describing the EHV Technology Program.

7. Miscellaneous Support Activities

This task involves various "quick-support" activities requested by the $R$ \& $D$ Coordinating Committee. One potential task is the preparation of another film on the Near-term Electric Vehicle program. Other activities in this area will be assigned as they occur during the year. 
2.5.2 Electric and Hybrid Vehicle Analysis Support (JO 7766)

This task area encompasses assignments of analysis and assessment of electric and hybrid vehicle systems and components. Assignments as of November 6, 1978 include:

1. Planning Grant Technical Analyses

Technical analyses of the next round of Planning Grant applications are the scope of this task.

2. Loan Guaranty Technical Analyses

It is anticipated that we will be requested to provide technical analyses and evaluations of applications for loan guarantees.

3. Proposal/Report/Correspondence Analyses

This is an expansion of work in the preparation of replies to incoming correspondence. This will include more detailed analyses of proposals and reports, reviews of the DOE EHV Annual and Quarterly Reports, and reviews of miscellaneous DOE reports.

4. Purdue Resource Allocation Model

This task entails critiquing and assisting in the subsequent implementation of the Purdue University Opportunity/Risk Assessment Study.

5. Corporate Average Fuel Economy Analysis

It is anticipated that this analytical study will continue and expand during the year as Congressional hearings are conducted and legislative proposals are offered.

2.5.3 Electric and Hybrid Vehicle Performance Standards Support (JO 7767)

This task involves analyses of the current state of the art of the performance capabilities of EHV technology to provide a basis for recommending future-year EHV performance standards. It will provide DOE with a defensible basis for setting future standards. The first cycle is to be completed by December 1979 .

2.6 Technology Assessment and Implementation (JO 7768)

This JO is reserved for prospective assignments of analysis and assessment support for the Technology Assessment and Implementation Branch. This Branch has not committed funds to the contract; no assignments have yet been requested. 
$2.7 \quad$ Technical Program Documentation

2.7.1 Program Planning and Nnalysis (JO 7769)

This JO is reserved for prospective assignments of analysis and planning support for TEC division-wide activities or for those planning activities which overlap Branch functions. No assignments have yet been made.

2.7.2 Management Review and Control Documentation (JO 7770)

This JO is reserved for prospective assignments of planning and documentation support for preparation of TEC's annual MRCD. No assignments have yet been made.

3. Reporting and Coordination

As can be noted from the discussion in Section 2 above, the preponderance of assignments involves technical analysis support of the quick-response, relatively short-duration nature. These work efforts are under way simultaneously for five Branches of TEC, and the results are often incorporated into ongoing TEC activities, as completed. The principal reporting mechanism from Aerospace to TEC pertaining to the analysis results of a given effort is a "memorandum report" transmitted by letter from Aerospace to TEC; the transmittal letter bears an identification number which incorporates the JO under which the work was performed.

In cases where the end product is more extensive, it may be published as an Aerospace technical report or as a DOE/TEC report, depending upon the wishes of the cognizant branch chief.

A Monthly Progress Report (MPR) is the principal tool for tracking (1) the tasks assigned to Aerospace, (2) the status and progress made in each such assignment, and (3) the expenditure of funds in each task area. For historical and record-keeping purposes, each such report will include an Appendix of Assignments and an Appendix of Reporting (see Appendix B of this report). In this manner, each branch chief can easily track assignments made and results received. In the expenditure area, each MPR will contain a breakdown, by Jo, of both monthly and cumulative expenditures to date. 
Summary: Table of Principal Reporting Activities of

The Aerospace Corporation under Contract No. EM-78-C-03-2184

(Includes formal reports, drafts, and memorandums or other

working Japers transmitted to DOE/TEC personnel to document progress on assigned task activities)

Period Through :31 March 1979

\begin{tabular}{|c|c|c|c|}
\hline $\begin{array}{l}\text { Job Order } \\
\text { Number }\end{array}$ & Description & $\begin{array}{l}\text { Type of } \\
\text { Report }\end{array}$ & $\begin{array}{l}\text { Recipient at } \\
\text { DOE/TEC }\end{array}$ \\
\hline \multirow[t]{7}{*}{7751} & Automotive Technology Assessment Study & & \\
\hline & $\begin{array}{l}\text { 1. Comments and Suggestions Pertaining to Format and } \\
\text { Content Development of First Annual Report to Congress } \\
\text { (Title III of Public Law } 95-238 \text { ) }\end{array}$ & $\begin{array}{l}\text { Briefing ( } 23 \text { pages) } \\
\text { to DOE/NASA Man- } \\
\text { agement Review } \\
\text { Meeting, } 6-27-78\end{array}$ & $\begin{array}{l}\text { Lombardi, } \\
\text { et al. }\end{array}$ \\
\hline & $\begin{array}{l}\text { 2. First "Strawman" Rough Draft of Annual Report to Congress } \\
\text { on Implementation of Public Law } 95-238\end{array}$ & $\begin{array}{l}\text { Draft report, } \\
7751.78 \text {.MGH- } 222 \\
1 \text { November } 1978\end{array}$ & Lombardi \\
\hline & $\begin{array}{l}\text { 3. Second "Strawman" Rough Draft of Annual Report to } \\
\text { Congress on Implementation of Public Law 95-238 }\end{array}$ & $\begin{array}{l}\text { Draft report } \\
7751.78 . \text { WUR. } 125 \\
20 \text { December } 1978\end{array}$ & Lombardi \\
\hline & $\begin{array}{l}\text { 4. Third Draft of Anmual Report to Congress on Implementa- } \\
\text { tion of Public La: } 95-238\end{array}$ & $\begin{array}{l}\text { Draft Report, } \\
7751.79 . \text { WUR.128 } \\
4 \text { January } 1979\end{array}$ & Lombardi \\
\hline & $\begin{array}{l}\text { 5. Fourth Draft of Annual Report to Congress on Implementa- } \\
\text { tion of Public Law } 95-238\end{array}$ & $\begin{array}{l}\text { Draft report } \\
7751.79 . \text { WUR.02 } \\
5 \text { January } 1979\end{array}$ & Lombardi \\
\hline & $\begin{array}{l}\text { 6. Fifth Draft of Annual Report to Congress on Implementa- } \\
\text { tion of Public Law } 95-238\end{array}$ & $\begin{array}{l}\text { Draft report, } \\
7751.79 \text {. WUR } .07 \\
29 \text { January } 1979\end{array}$ & Lombardi \\
\hline
\end{tabular}




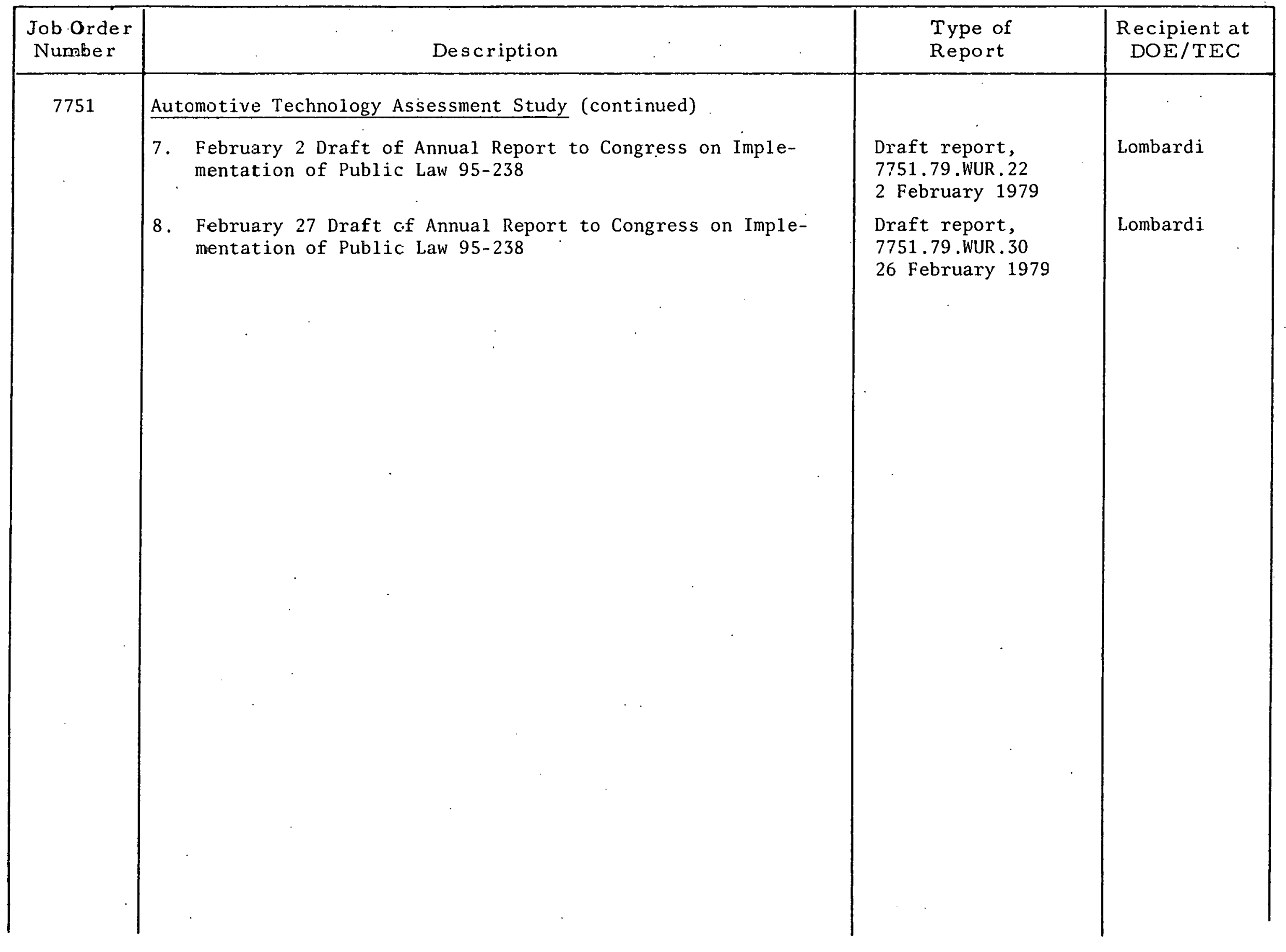




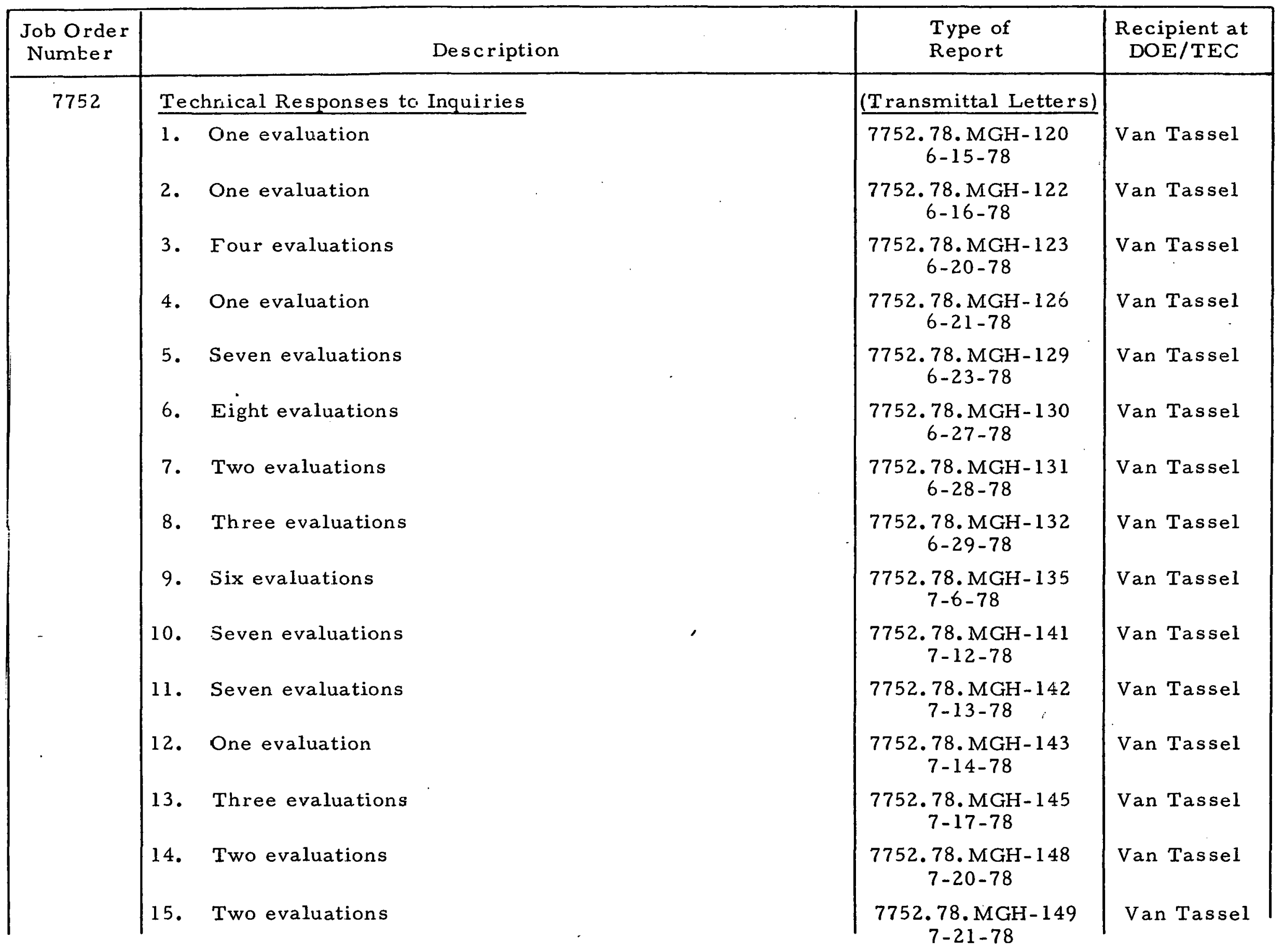




\begin{tabular}{|c|c|c|c|}
\hline $\begin{array}{l}\text { Job Order } \\
\text { Number }\end{array}$ & Description & $\begin{array}{l}\text { Type of } \\
\text { Report }\end{array}$ & $\begin{array}{l}\text { Recipient at } \\
\text { DOE/TEC }\end{array}$ \\
\hline \multirow[t]{16}{*}{7752} & Tecinical Responses to Inquiries (continued) & (Transmittal Letters) & \\
\hline & 16. Seven evaluations & $\begin{array}{c}\text { 7752. 78. MGH }-151 \\
7-25-78\end{array}$ & Van Tassel \\
\hline & 17. One evaluation & $\begin{array}{c}\text { 7752. } 78 . \mathrm{MGH}-152 \\
7-26-78\end{array}$ & Van Tassel \\
\hline & 18. Five evaluations & $\begin{array}{l}\text { 7752. 78. MGH }-154 \\
8-1-78\end{array}$ & Van Tassel \\
\hline & 19. Two evaluations & $\begin{array}{c}\text { 7752. 78. MGH }-155 \\
8-3-78\end{array}$ & Van Tassel \\
\hline & 20. One evaluation & $\begin{array}{c}\text { 7752. } 78 . \mathrm{MGH}-156 \\
8-4-78\end{array}$ & Van Tassel \\
\hline & 21. Two evaluations & $\begin{array}{c}\text { 7752. 78. MGH- } 159 \\
8-9-78\end{array}$ & Van Tassel \\
\hline & 22. Six evaluations & $\begin{array}{c}\text { 7752. 78. MGH- } 165 \\
8-15-78\end{array}$ & Van Tassel \\
\hline & 23. One evaluation & $\begin{array}{c}\text { 7752. 78. MGH }-169 \\
8-17-78\end{array}$ & Van Tassel \\
\hline & 24. Four evaluations & $\begin{array}{c}\text { 7752. 78. MGH }-175 \\
8-21-78\end{array}$ & Van Tassel \\
\hline & 25. Two evaluations & $\begin{array}{c}\text { 7752. 78. MGH }-176 \\
8-22-78\end{array}$ & Van Tassel \\
\hline & 26. Three evaluations & $\begin{array}{c}\text { 7752. 78. MGH }-183 \\
8-25-78\end{array}$ & Van Tassel \\
\hline & 27. Two evaluations & $\begin{array}{c}\text { 7752. 78. MGH }-187 \\
8-30-78\end{array}$ & Van Tassel \\
\hline & 28. Two evaluations & $\begin{array}{c}\text { 7752. } 78 . \mathrm{MGH}-188 \\
8-31-78\end{array}$ & Van Tassel \\
\hline & 29. Two evaluations & $\begin{array}{c}7752.78 . \mathrm{MGH}-194 \\
9-8-70\end{array}$ & Van Tasse1 \\
\hline & 30. Two evaluations & 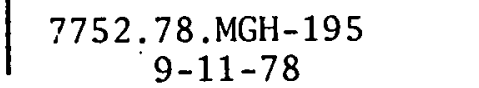 & Van Tassel \\
\hline
\end{tabular}




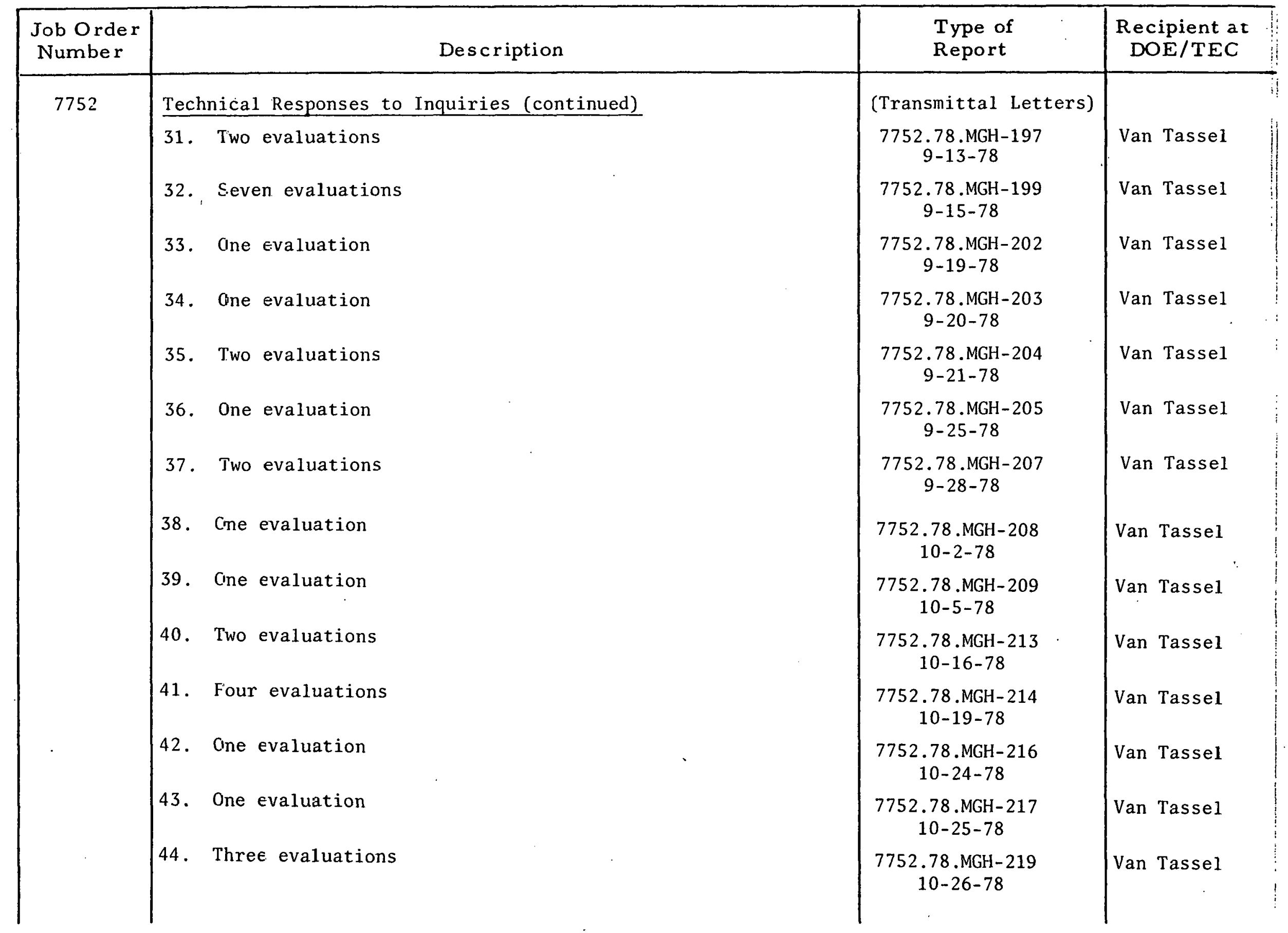




\begin{tabular}{|c|c|c|c|}
\hline $\begin{array}{l}\text { Job Order } \\
\text { Number }\end{array}$ & Description & $\begin{array}{l}\text { Type of } \\
\text { Report }\end{array}$ & $\begin{array}{l}\text { Recipient at } \\
\text { DOE/TEC }\end{array}$ \\
\hline \multirow[t]{14}{*}{7752} & Technical Responses to Inquiries (continued) & & \\
\hline & 45. Three evaluations & $\begin{array}{c}7752.78 . \mathrm{MGH}-220 \\
10-27-78\end{array}$ & Van Tassel \\
\hline & 46. One evaluation & $\begin{array}{c}7752.78 . \mathrm{MGH}-221 \\
10-31-78\end{array}$ & Van Tassel \\
\hline & 47. Three evaluations & $\begin{array}{c}7752.78 . \mathrm{MGH}-223 \\
11-2-78\end{array}$ & Van Tassell \\
\hline & 48. One evaluation & $\begin{array}{c}7752.78 . \mathrm{MGH}-235 \\
11-17-78\end{array}$ & Van Tassell \\
\hline & 49. One evaluation & $\begin{array}{c}7752.78 . \mathrm{MGH}-236 \\
11-20-78\end{array}$ & Van Tassell \\
\hline & 50. Three evaluations & $\begin{array}{c}7752.78 . \mathrm{MGH}-241 \\
11-30-78\end{array}$ & Van Tasse11 \\
\hline & 51. Two evaluations & $\begin{array}{c}7752.78 . \mathrm{MGH}-243 \\
11-30-78\end{array}$ & Van Tassell \\
\hline & 52. One evaluation & $\begin{array}{c}7752.78 . \mathrm{MGH}-244 \\
12-5-78\end{array}$ & Van Tassel \\
\hline & 53. Five evaluations & $\begin{array}{c}7752.78 . \mathrm{MGH}-251 \\
12-12-78\end{array}$ & Van Tasse 1 \\
\hline & 54. Dne evaluation & $\begin{array}{c}7752.78 . \mathrm{MGH}-255 \\
12-20-78\end{array}$ & Van Tasse1 \\
\hline & 55. Dne evaluation & $\begin{array}{c}7752.78 . \mathrm{MGH}-256 \\
12-26-78\end{array}$ & Van Tassel \\
\hline & 56. Three evaluations & $\begin{array}{c}7752.78 . \mathrm{MGH}-257 \\
12-27-78\end{array}$ & Van Tasse1 \\
\hline & $\begin{array}{l}\text { Noie: Commencing in January } 1979 \text {, all evaluations were } \\
\text { telefaxed directly from Los Angeles to Germantown, } \\
\text { Maryland, as received. }\end{array}$ & & \\
\hline
\end{tabular}




\begin{tabular}{|c|c|c|c|}
\hline $\begin{array}{l}\text { Job Order } \\
\text { Numier }\end{array}$ & Description & $\begin{array}{l}\text { Type of } \\
\text { Report }\end{array}$ & $\begin{array}{l}\text { Recipient at } \\
\text { DOE/TEC }\end{array}$ \\
\hline \multirow[t]{11}{*}{7753} & Highway Systems Analysis Support & & \\
\hline & $\begin{array}{l}\text { 1. Review of SRI Draft Report, "Diesel Cars in the } \\
\text { United States" }\end{array}$ & $\begin{array}{l}\text { Memorandum Report } \\
7753.78 \text {. WUR. } 66 \\
7-11-78\end{array}$ & Maxfield \\
\hline & $\begin{array}{l}\text { 2. Test Plan for Demonstration and Evaluation of Gas } \\
\text { Turbine Engine Powered Automobiles }\end{array}$ & $\begin{array}{l}\text { Memorandum Report } \\
7753.78 \text {. WUR. } 79 \\
7-14-78\end{array}$ & Themak \\
\hline & $\begin{array}{l}\text { 3. Variables to Consider in Auto Fuel Consumption } \\
\text { Projections }\end{array}$ & $\begin{array}{l}\text { Memorandum Report } \\
7753.78 . \mathrm{MGH}-144 \\
7-14-78\end{array}$ & Thur \\
\hline & $\begin{array}{l}\text { 4. Review of Proposal, "Automobile Fuel Injection } \\
\text { Device" }\end{array}$ & $\begin{array}{l}\text { Memorandum Report } \\
7753.78 . \text { WCG }-18 \\
7-17-78\end{array}$ & Lombardi \\
\hline & 5. Review of Proposal, "The Otto-Atkinson Engine" & $\begin{array}{l}\text { Memorandum Report } \\
7753.78 . \text { WCG }-20 \\
7-18-78\end{array}$ & Lombardi \\
\hline & $\begin{array}{l}\text { 6. Review of Proposal, "Development and Demonstration } \\
\text { of Medium Duty Trucks Powered by Open Chamber } \\
\text { Stratified Charge Engines" }\end{array}$ & $\begin{array}{l}\text { Memorandum Report } \\
\text { 7753.78. MGH }-157 \\
8-7-78\end{array}$ & Themak \\
\hline & $\begin{array}{l}\text { 7. Diesel Engine Research and Development Status, } \\
\text { and Requirements }\end{array}$ & $\begin{array}{l}\text { Briefing (40 pages) } \\
\text { to DOE/TEC in } \\
\text { Washington, D. C. } \\
\qquad 8-11-78\end{array}$ & $\begin{array}{l}\text { Brogan, } \\
\text { et al. }\end{array}$ \\
\hline & $\begin{array}{l}\text { 8. Review of Propcsal, "Hydraulic Power Management } \\
\text { System for Automotive Applications" }\end{array}$ & $\begin{array}{c}\text { Memorandum Report } \\
\text { 7753.78. WCG-23 } \\
8-15-78\end{array}$ & Themak \\
\hline & $\begin{array}{l}\text { 9. "DOE Comments on the Prospective DOT } 1979 \\
\text { Report to Congress"' (First Draft) }\end{array}$ & $\begin{array}{c}\text { Memorandum Report } \\
7753.78 . \text { MGH }-168 \\
8-16-78\end{array}$ & Lombardi \\
\hline & $\begin{array}{l}\text { 10. Review of Propasal, "Improvement of Automotive } \\
\text { Coolant Pump Performance" }\end{array}$ & $\begin{array}{c}\text { Memorandum Report } \\
7753.78 . \text { WCG }-24 \\
8-18-78\end{array}$ & Themak \\
\hline
\end{tabular}




\begin{tabular}{|c|c|c|c|}
\hline $\begin{array}{l}\text { Job Order } \\
\text { Number }\end{array}$ & Description & $\begin{array}{l}\text { Type of } \\
\text { Report }\end{array}$ & $\begin{array}{l}\text { Recipient at } \\
\text { DOE/TEC }\end{array}$ \\
\hline \multirow[t]{12}{*}{7753} & Highway Systems Analysis Support (continued) & & \\
\hline & $\begin{array}{l}\text { 11. "DOE Comments on the Prospective DOT } 1979 \text { Report } \\
\text { to Congress" (First Draft of 34-page Briefing) }\end{array}$ & $\begin{array}{l}\text { Memorandum Report } \\
7753.78 . \mathrm{MGH}-186 \\
8-29-78\end{array}$ & Lombardi \\
\hline & $\begin{array}{l}\text { 12. Final Draft of Briefing, "DOE Comments on the Prospective } \\
\text { DOT } 1979 \text { Report to Congress" ( } 34 \text { pages) }\end{array}$ & $\begin{array}{l}\text { Briefing on Sept. } 13 \\
1978 \text { at DOT Hdqrtrs. }\end{array}$ & $\begin{array}{l}\text { Lombardi, } \\
\text { Thur, Brogan }\end{array}$ \\
\hline & $\begin{array}{l}\text { 13. Final Draft of } 85 \text {-page document, "DOE Comments on the } \\
\text { Prospective DOT } 1979 \text { Report to Congress" }\end{array}$ & $\begin{array}{l}25 \text { copies delivered } \\
\text { on Sept. } 13,1978\end{array}$ & Lombardi \\
\hline & $\begin{array}{l}\text { 14. Review of Proposal, "Mini-Sam Propulsion System," } \\
\text { I.D. No. THP } 7800838\end{array}$ & $\begin{array}{l}\text { Memorandum Report } \\
7753.78 . \text { WCG.27 } \\
1 \text { September } 1978\end{array}$ & Lombardi \\
\hline & $\begin{array}{l}\text { 15. Rebuttal to Proposal Evaluation, "Britalus Continuous } \\
\text { Combustion Rotary Engine" }\end{array}$ & $\begin{array}{l}\text { Memorandum Report, } \\
7753.78 . \text { WCG. } 28 \\
4 \text { September } 1978\end{array}$ & Lombardi \\
\hline & 16. Review of Proposal, "Fuel Injection Systems" & $\begin{array}{l}\text { Memorandum Report, } \\
7753.78 . \text { WCG. } 29 \\
7 \text { September } 1978\end{array}$ & Themak \\
\hline & $\begin{array}{l}\text { 17. Review of Proposal, "Orbital Hydrostatic Accumulating } \\
\text { Drive" }\end{array}$ & $\begin{array}{l}\text { Memorandum Report, } \\
7753.78 . \text { WCG. } 30 \\
11 \text { September } 1978\end{array}$ & Lombardi \\
\hline & $\begin{array}{l}\text { 18. Review of Proposal, "Ceramic Helical Expanders for } \\
\text { Brayton Cycle Heat Engines" }\end{array}$ & $\begin{array}{l}\text { Memorandum Report, } \\
7753.78 . \text { RRB.02, } \\
15 \text { September } 1978\end{array}$ & Thur \\
\hline & 19. Review of Proposal, "Fuel Savings Project" & $\begin{array}{l}\text { Memorandum Report, } \\
7753.78 . \text { WCG. } 31 \\
18 \text { September } 1978\end{array}$ & Lombardi \\
\hline & 20. Review of Proposal, "Brinkerhoff Split Cycle Engine" & $\begin{array}{l}\text { Memorandum Report, } \\
7753.78 . \text { RRB .03, } \\
25 \text { September } 1978\end{array}$ & Themak \\
\hline & $\begin{array}{l}\text { 21. First draft, "Diesel Engine Research and Development } \\
\text { Status and Needs" }\end{array}$ & $\begin{array}{l}\text { Memorandum Report, } \\
7753.78 . \text { WUR.102, } \\
11 \text { September } 1978\end{array}$ & Brogan \\
\hline
\end{tabular}




\begin{tabular}{|c|c|c|c|}
\hline $\begin{array}{l}\text { Job Order } \\
\text { Number }\end{array}$ & Description & $\begin{array}{l}\text { Type of } \\
\text { Report }\end{array}$ & $\begin{array}{l}\text { Recipient at } \\
\text { DOE/TEC }\end{array}$ \\
\hline \multirow[t]{12}{*}{7753} & Highway Systems Analysis Support (continued) & & \\
\hline & $\begin{array}{l}\text { 32. Review of Proposal, "A Reciprocating Brayton Cycle } \\
\text { Engine" (Warren Engine) }\end{array}$ & $\begin{array}{l}\text { Memorandum Report, } \\
7753.78 . \text { WCG. } 35 \\
16 \text { November } 1978\end{array}$ & Lombardi \\
\hline & $\begin{array}{l}\text { 33. Final Report, "Diesel Engine Research and Development } \\
\text { Status and Needs" }\end{array}$ & $\begin{array}{l}\text { Aerospace Report No. } \\
\text { ATR } 78(7753)-1 \\
\text { September } 1978\end{array}$ & Brogan, et al \\
\hline & $\begin{array}{l}\text { 34. Review of Proposal, "Synthetic Atmosphere Engine } \\
\text { Development," by S. C. Plotkin }\end{array}$ & $\begin{array}{l}\text { Memorandum Report, } \\
7753.78 . \text { WUR } .123 \\
7 \text { December } 1978\end{array}$ & Thur \\
\hline & 35. Review of Proposal, "Adkins Rotary Engine" & $\begin{array}{l}\text { Memorandum Report } \\
7753.78 . \text { GJM. } 32 \\
7 \text { December } 1978\end{array}$ & Lombardi \\
\hline & $\begin{array}{l}\text { 36. Review of Proposal, "Design and Development of Improved } \\
\text { Stirling Cycle Engine," by Don S. Slack } \\
\text { (THP-7900091) }\end{array}$ & $\begin{array}{l}\text { Memorandum Report } \\
7753.78 . \text { RBB } .10 \\
19 \text { December } 1978\end{array}$ & Thur \\
\hline & 37. Transmittal of Changes to Diesel Report & $\begin{array}{l}\text { Memorandum } \\
7753.78 . \text { WUR } .122 \\
6 \text { December } 1978\end{array}$ & Lombardi \\
\hline & $\begin{array}{l}\text { 38. Transmittal of Masters for Diesel Report, } \\
\text { ATR-78(7753)-1 }\end{array}$ & $\begin{array}{l}\text { Memorandum } \\
7753.78 . \text { WUR } .127 \\
21 \text { December } 1978\end{array}$ & Lombardi \\
\hline & $\begin{array}{l}\text { 39. Review of Proposal, "Latent Heat Re-use System for } \\
\text { Steam Engines," by Earling Steiner (THP-7900076) }\end{array}$ & $\begin{array}{l}\text { Memorandum Report } \\
7753.78 . \text { WCG } .38 \\
29 \text { December } 1978\end{array}$ & Lombardi \\
\hline & $\begin{array}{l}\text { 40. Masters and six copies of report, "Fuel Consumption and } \\
\text { Engine Horsepower Projections for Highway Transportation } \\
\text { Sector" }\end{array}$ & $\begin{array}{l}\text { Aerospace Report No. } \\
\text { ATR }-78(7753)-2 \\
\text { April } 1978\end{array}$ & Lombardi \\
\hline & $\begin{array}{l}\text { 41. Review of Proposa1, "Determination of Two Phase Turbine } \\
\text { Engine Characteristics" (TNP 7900261 }\end{array}$ & $\begin{array}{l}\text { Memorandum Report } \\
7753.79 . \text { WCG } .02 \\
15 \text { January } 1979\end{array}$ & Lombardi \\
\hline & 42. Draft inputs to DOE's Diesel Briefing Book & $\begin{array}{l}\text { Memorandum Report } \\
7753: 79 \text {. WUR } 06 \\
22 \text { January } 1979\end{array}$ & Lombardi \\
\hline
\end{tabular}




\begin{tabular}{|c|c|c|c|}
\hline $\begin{array}{l}\text { Job Order } \\
\text { Number }\end{array}$ & Description & $\begin{array}{l}\text { Type of } \\
\text { Report }\end{array}$ & $\begin{array}{c}\text { Recipient at } \\
\text { DOE/TEC }\end{array}$ \\
\hline \multirow[t]{10}{*}{7753} & \multicolumn{3}{|l|}{ Highway Systems Analysis Support (continued) } \\
\hline & $\begin{array}{l}\text { 43. Review of "Passənger Automobile Weight Projections of } \\
\text { 1983-1986" (Corporate-Tech Planning, Inc.) }\end{array}$ & $\begin{array}{l}\text { Memorandum Report } \\
7753.79 . J J D .04 \\
29 \text { January } 1979\end{array}$ & Lombardi \\
\hline & $\begin{array}{l}\text { 44. Rəview of "Documentation of Fuel Economy and Cost } \\
\text { Estimates Used in the Analysis of NEP II Automotive } \\
\text { Conservation Ir:itiatives" (Energy and Environmental } \\
\text { Analysis, Inc.) }\end{array}$ & $\begin{array}{l}\text { Memorandum Report } \\
7753 . \text { WUR. } 79.31 \\
26 \text { February } 1979\end{array}$ & Lombardi \\
\hline & $\begin{array}{l}\text { 45. Review of "EPA Draft Regulatory Analysis Report for } \\
\text { Díesel Technolozy and Cost" }\end{array}$ & $\begin{array}{l}\text { Memorandum Report } \\
7753 . \text { WUR } .79 .33 \\
2 \text { March } 1979\end{array}$ & Themak \\
\hline & 46. Review of Proposa1, "Gearbine Rotary Engine" & $\begin{array}{l}\text { Memorandum Report } \\
7753, \text { RBB } 79.05 \\
8 \text { March } 1979\end{array}$ & Lombardi \\
\hline & $\begin{array}{l}\text { 47. Draft response to Congressman Findley regarding } \\
\text { carburetor float issue }\end{array}$ & $\begin{array}{l}\text { Memorandum Report } \\
7753.79 . \text { WCG.09 } \\
23 \text { March } 1979\end{array}$ & Lombardi \\
\hline & $\begin{array}{l}\text { 48. Review of Proposal, "Dual-Cycle Heat Engine," submitted } \\
\text { by David M. Van Den Einde }\end{array}$ & $\begin{array}{l}\text { Memorandum Report } \\
7753.79 . \text { RBB } 07 \\
26 \text { March } 1979\end{array}$ & Lombardi \\
\hline & 49. Review of Proposal, "Pentagonal Electromagnetic Engine" & $\begin{array}{l}\text { Memorandum Report } \\
7753.79 \text {.WCG.11 } \\
28 \text { March } 1979\end{array}$ & Lombardi \\
\hline & $\begin{array}{l}\text { 50. "Alternate Fuel Economy Measures and Transportation } \\
\text { Mileage Optimization" }\end{array}$ & $\begin{array}{l}\text { Memorandum Report } \\
7753.79 . \text { WUR. } 34 \\
14 \text { March } 1979\end{array}$ & Brogan \\
\hline & 51. Crude Oil Savings (in Bar Chart format) & $\begin{array}{l}\text { FAXED Chart } \\
23 \text { March } 1979\end{array}$ & Brogan \\
\hline
\end{tabular}




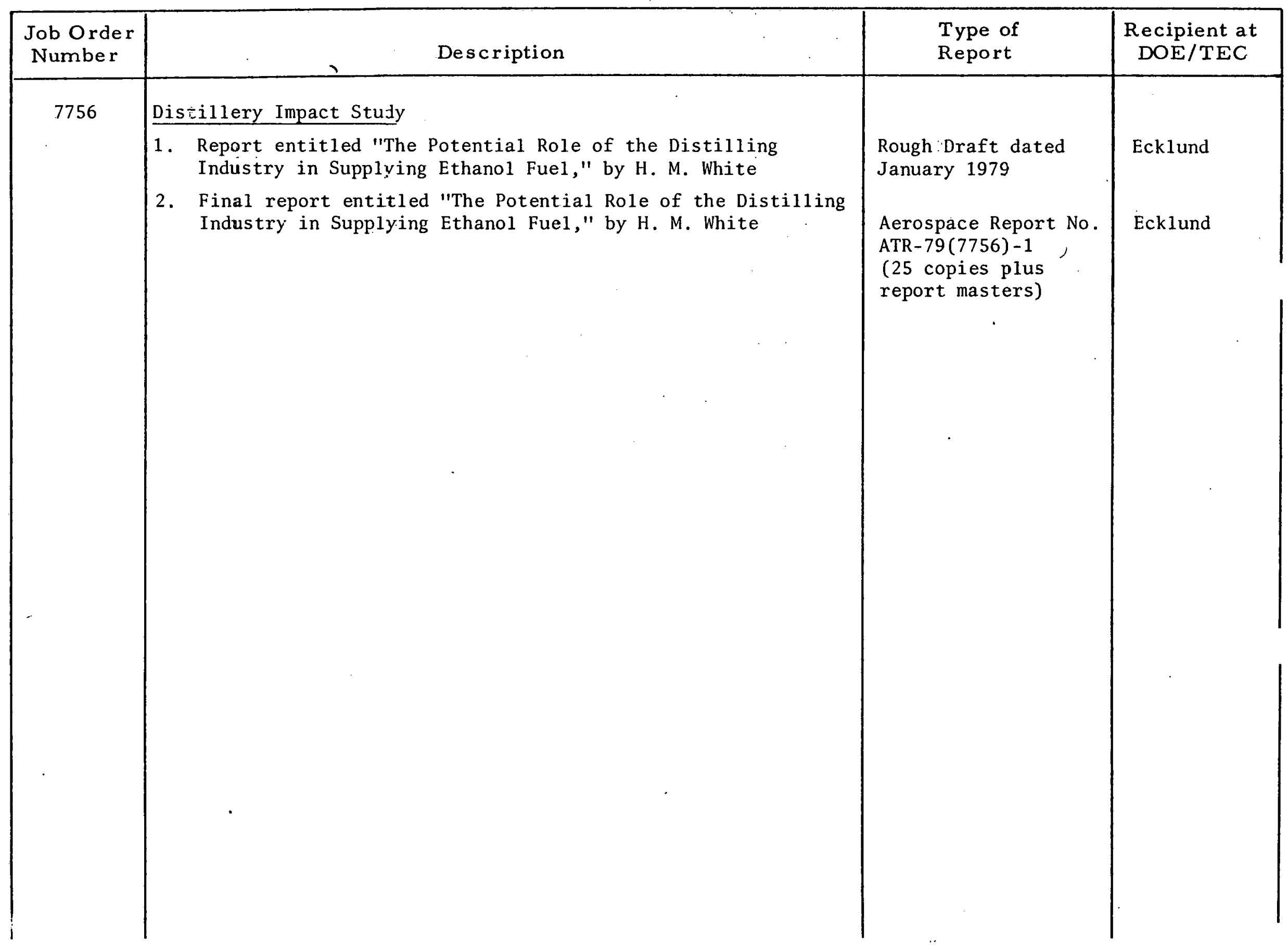




\begin{tabular}{|c|c|c|c|}
\hline $\begin{array}{l}\text { Job Order } \\
\text { Number }\end{array}$ & Description & $\begin{array}{l}\text { Type of } \\
\text { Report }\end{array}$ & $\begin{array}{c}\text { Recipient at } \\
\text { DOE/TEC }\end{array}$ \\
\hline 7757 & $\begin{array}{l}\text { Immediate Action Stucies Support } \\
\text { 1. Typewritten drafts of various sections of report for } \\
\text { End-use Subcommittee of the Alcohol Fuels Policy Review } \\
\text { during period August } 7,1978 \text { to October 17, 1978 }\end{array}$ & $\begin{array}{l}\text { Memorandum of Record } \\
7757.78 \text {. MGH-237 } \\
27 \text { November } 1978 \\
\vdots \\
\vdots \\
\vdots \\
\end{array}$ & Ecklund \\
\hline
\end{tabular}




\begin{tabular}{|c|c|c|c|}
\hline $\begin{array}{l}\text { Job Order } \\
\text { Number }\end{array}$ & Description & $\begin{array}{l}\text { Type of } \\
\text { Report }\end{array}$ & $\begin{array}{c}\text { Recipient at } \\
\text { DOE/TEC }\end{array}$ \\
\hline 7758 & $\begin{array}{l}\text { Fuel Economy Improvement Potential Study } \\
\text { 1. Draft of "Effect of Combustion Burn Time on the Efficiency } \\
\text { of Spark Ignition Engines" }\end{array}$ & $\begin{array}{l}\text { Draft Report, } \\
7758.79 \text {.GJM.03 } \\
9 \text { February } 1979\end{array}$ & Fleming \\
\hline
\end{tabular}




\begin{tabular}{|c|c|c|c|}
\hline $\begin{array}{l}\text { Job Order } \\
\text { Number }\end{array}$ & Description & $\begin{array}{l}\text { Type of } \\
\text { Report }\end{array}$ & $\begin{array}{l}\text { Recipient at } \\
\text { DOE/TEC }\end{array}$ \\
\hline \multirow[t]{10}{*}{7759} & Alternative Fuels Analysis Support & & \\
\hline & $\begin{array}{l}\text { 1. Review of LLL Report, "Energy Storage Systems } \\
\text { for Automobile Propulsion" }\end{array}$ & $\begin{array}{c}\text { Memorandum Report } \\
7759.78 . \text { JJD. } 17 \\
7-17-78\end{array}$ & Ecklund \\
\hline & $\begin{array}{l}\text { 2. Project Plan for Reliability Fleet Testing of } \\
\text { Alcohol/Gasoline Blends (First Draft) }\end{array}$ & $\begin{array}{l}\text { Memorandum Report } \\
\text { 7759.78. MGH -153 } \\
7-31-78\end{array}$ & Ecklund \\
\hline & $\begin{array}{l}\text { 3. Detailed Plans for First Year's Activities of } \\
\text { Reliability Fleet Test Program }\end{array}$ & $\begin{array}{l}\text { Memorandum Report, } \\
7759.78 . \text { MGH- } 232 \\
14 \text { November } 1978\end{array}$ & Ecklund \\
\hline & $\begin{array}{l}\text { 4. Iniated Efforts to Secure Services of SWRI to Assist in } \\
\text { Reliability Fleet Test Planning }\end{array}$ & $\begin{array}{l}\text { Memorandum } \\
7759.78 . \text { MGH }-248 \\
11 \text { December } 1978\end{array}$ & Ecklund \\
\hline & $\begin{array}{l}\text { 5. Submitted paper, "Hydrogen Powered Versus Battery } \\
\text { Powered Automobiles," for publication to International } \\
\text { Journal of Hydrogen Energy }\end{array}$ & $\begin{array}{l}\text { Memorandum Report } \\
7759.78 . J J D .38 \\
14 \text { December } 1978\end{array}$ & $\begin{array}{l}\text { Nejat } \\
\text { Veziroglu }\end{array}$ \\
\hline & 6. Report of CRC Meeting in Detroit on 12 December 1978 & $\begin{array}{l}\text { H. White memo, } \\
14 \text { December } 1978\end{array}$ & Ecklund \\
\hline & 7. Report of Visit to Southwestern Bell and BETC & $\begin{array}{l}\text { Memorandum } \\
7759.78 . \mathrm{HMW} \\
28 \text { December } 1978\end{array}$ & Ecklund \\
\hline & $\begin{array}{l}\text { 8. Updated Project Plan for Reliability Fleet Testing of } \\
\text { Alcohol/Gasoline Blends }\end{array}$ & $\begin{array}{l}\text { Document dated } \\
30 \text { March } 1979\end{array}$ & Ecklund \\
\hline & $\begin{array}{l}\text { 9. Briefing package, "DOE Reliability Fleet Test Program } \\
\text { for Alcohol/Gasoline Fuels" (for presentation to } \\
\text { Contractors Coordination Meeting, April 24-26, 1979, } \\
\text { Dearborn, Michigan). }\end{array}$ & $\begin{array}{l}300 \text { copies of } \\
\text { Briefing Book }\end{array}$ & Ecklund \\
\hline
\end{tabular}




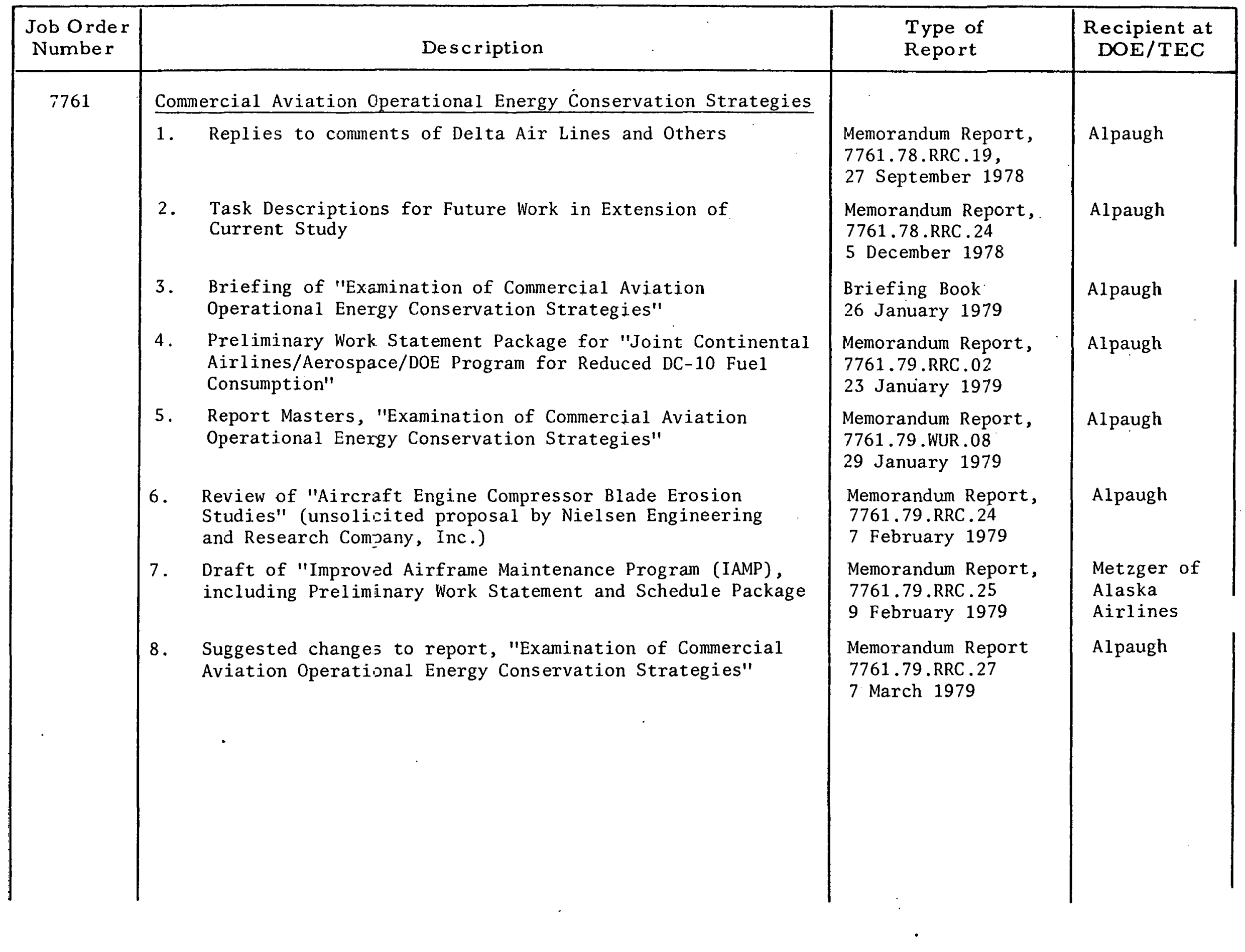




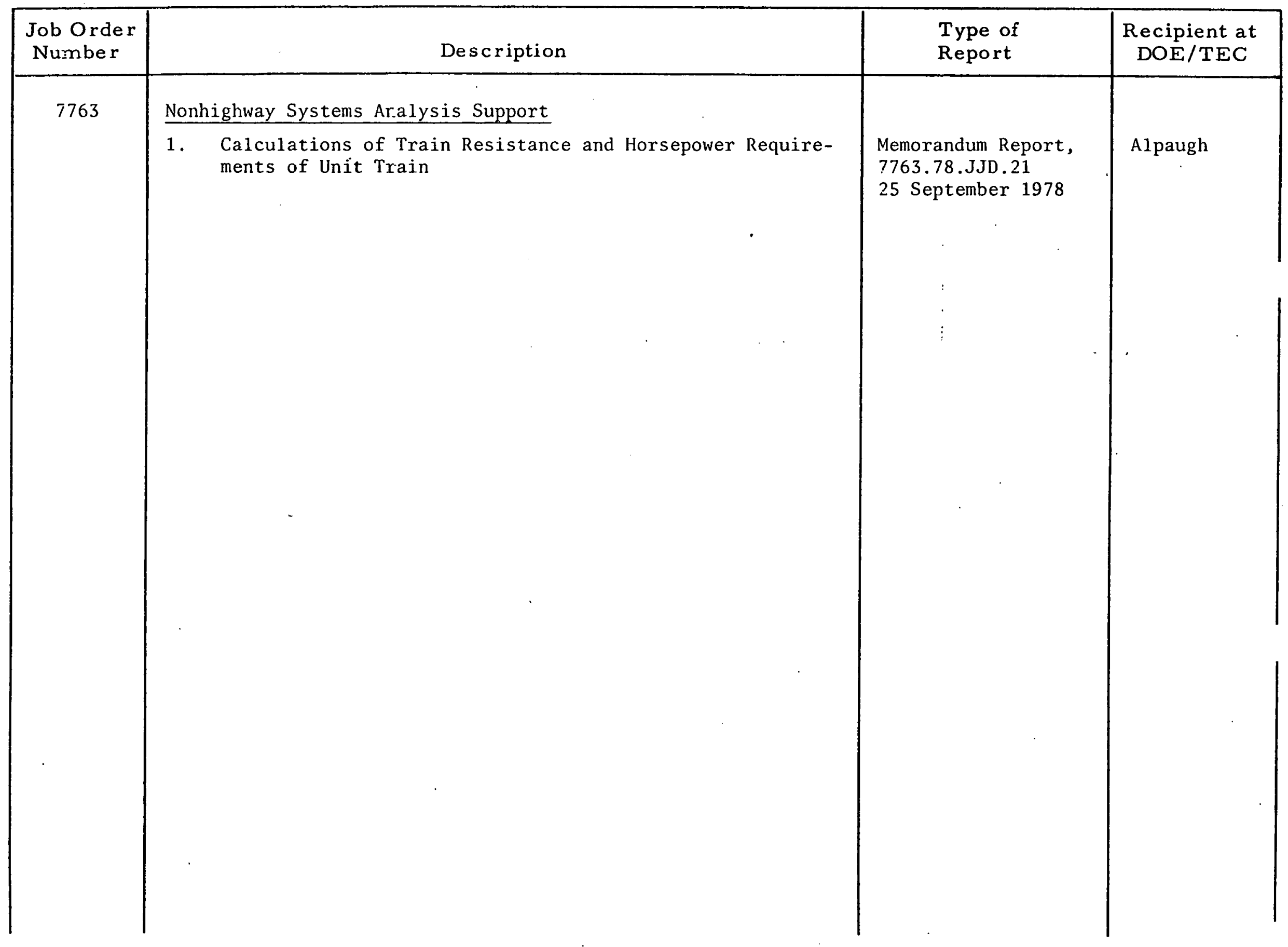




\begin{tabular}{|c|c|c|c|}
\hline $\begin{array}{l}\text { Job Order } \\
\text { Number }\end{array}$ & Description & $\begin{array}{l}\text { Type of } \\
\text { Report }\end{array}$ & $\begin{array}{l}\text { Recipient at } \\
\text { DOE/TEC }\end{array}$ \\
\hline \multirow[t]{9}{*}{7764} & New Concepts Evaliation (Proposals) & & \\
\hline & $\begin{array}{l}\text { 1. "Vehicle Drive Systems Including a Phased Rotary } \\
\text { Type Engine with an Infinitely Variable Gear Ratio } \\
\text { Automatic Trarsmission" (TNP-7800608) }\end{array}$ & $\begin{array}{l}\text { Memorandum Report } \\
7764.78 . \text { WCG }-16 \\
6-20-78\end{array}$ & Starr \\
\hline & $\begin{array}{l}\text { 2. "Development of the Wall The rmodynamic Cycle" } \\
\text { (THK-80014) }\end{array}$ & $\begin{array}{l}\text { Memorandum Report } \\
7764.78 . \text { WCG }-17 \\
6-23-78\end{array}$ & Sta $\mathbf{r}$ \\
\hline & $\begin{array}{l}\text { 3. "Research and Development of Concept Leading to an } \\
\text { Automotive Alternator with Significantly Improved } \\
\text { Efficiency and Weight" (TNP-7800582 }\end{array}$ & $\begin{array}{l}\text { Memorandum Report } \\
7764.78 \text {. WCG }-22 \\
8-3-78\end{array}$ & Star r \\
\hline & $\begin{array}{l}\text { 4. "An Energy Conserving Pollution Free Automobile } \\
\text { Exhaust Device" (TNP-780074) }\end{array}$ & $\begin{array}{l}\text { Memorandum Report } \\
\text { 7764. } 78 . \text { WCG }-25 \\
\quad 8-14-78\end{array}$ & Star $\mathbf{r}$ \\
\hline & $\begin{array}{l}\text { 5. "A Study of a Gasoline Economy Car of Unique Design" } \\
\text { (THP-7801104) }\end{array}$ & $\begin{array}{c}\text { Memorandum Report, } \\
7764.78 . \text { WCG } .33 \\
9-13-78\end{array}$ & Starr \\
\hline & 6. "Graybill 0il Rectifier" & $\begin{array}{l}\text { Memorandum Report, } \\
7764.78 . \text { GJM- } 28 \\
28 \text { November } 1978\end{array}$ & Starr \\
\hline & $\begin{array}{l}\text { 7. "Diesel Pulsejet Engine for Pleasure Boats," } \\
\text { (TNP-7900143) }\end{array}$ & $\begin{array}{l}\text { Memorandum Report, } \\
7764.78 . \text { WCG } .37 \\
14 \text { December } 1978\end{array}$ & Starr \\
\hline & $\begin{array}{l}\text { 8. "Optimization o } \underset{i}{ } \text { Rail Car Structures for Energy } \\
\text { Conservation," A Rebuttal }\end{array}$ & $\begin{array}{l}\text { Memorandum Report } \\
7764.78 . \text { WUR } 124 \\
14 \text { December } 1978\end{array}$ & Alpaugh \\
\hline
\end{tabular}




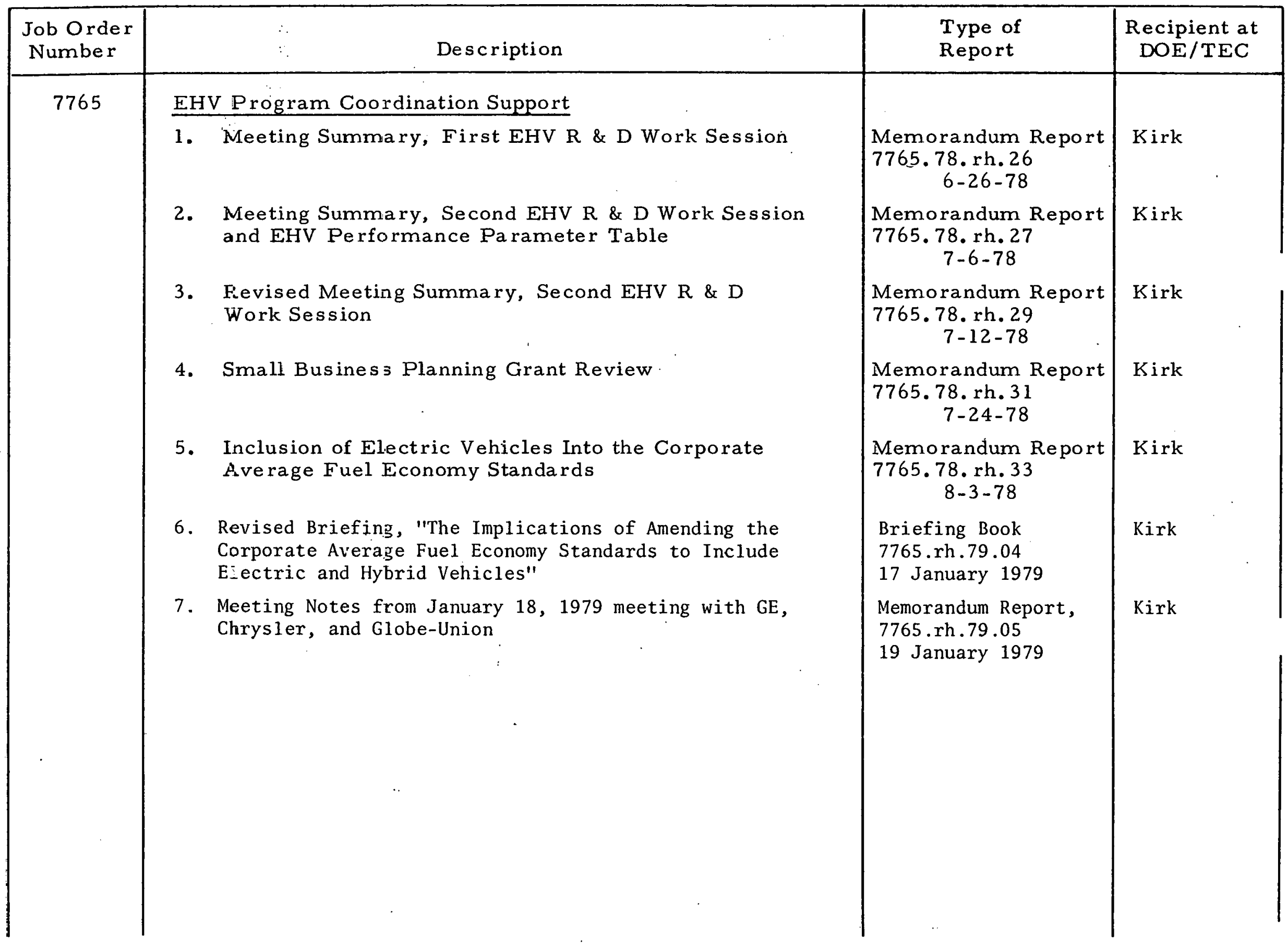




\begin{tabular}{|c|c|c|c|}
\hline $\begin{array}{l}\text { Jcb Order } \\
\text { Number }\end{array}$ & Description & $\begin{array}{l}\text { Type of } \\
\text { Report }\end{array}$ & $\begin{array}{l}\text { Recipient at } \\
\text { DOE/TEC }\end{array}$ \\
\hline 7770 & $\begin{array}{l}\text { Management Review and Control Documentation } \\
\begin{array}{l}\text { Revision of } 1978 \text { MRCD masters (originally prepared under } \\
\text { previous contract) }\end{array}\end{array}$ & $\begin{array}{l}\text { Memorandum Report, } \\
7770 . \text { WUR. } 78.103, \\
12 \text { Sept ember } 1978\end{array}$ & Wilson \\
\hline
\end{tabular}

